NASA Technical Memorandum 4481

\title{
Experimental and Computational Investigation of the NASA Low-Speed Centrifugal Compressor Flow Field
}

Michael D. Hathaway,

Randall M. Chriss, Jerry R. Wood, and Anthony J. Strazisar

September 1993 
NASA Technical Memorandum 4481

\title{
Experimental and Computational Investigation of the NASA Low-Speed Centrifugal Compressor Flow Field
}

\author{
Michael D. Hathaway \\ Vehicle Propulsion Directorate \\ U.S. Army Research Laboratory \\ Cleveland, Ohio 44135
}

and

Randall M. Chriss, Jerry R. Wood, and Anthony J. Strazisar

Lewis Research Center

Cleveland, Ohio

\section{N/SA}

National Aeronautics and

Space Administration

Office of Management

Scientific and Technical

Information Program 


\section{Summary}

An experimental and computational investigation of the NASA Lewis Research Center's low-speed centrifugal compressor (LSCC) flow field was conducted using laser anemometry and Dawes' three-dimensional viscous code. The experimental configuration consisted of a backswept impeller followed by a vaneless diffuser. Measurements of the three-dimensional velocity field were acquired at several measurement planes through the compressor. The measurements describe both the throughflow and secondary velocity field along each measurement plane. In several cases the measurements provide details of the flow within the blade boundary layers. Insight into the complex flow physics within centrifugal compressors is provided by the computational fluid dynamics analysis (CFD), and assessment of the CFD predictions is provided by comparison with the measurements. Five-hole probe and hot-wire surveys at the inlet and exit to the impeller as well as surface flow visualization along the impeller blade surfaces provided independent confirmation of the laser measurement technique. The results clearly document the development of the throughflow velocity wake that is characteristic of unshrouded centrifugal compressors.

\section{Introduction}

The complex curvature of centrifugal compressor impeller blade channels coupled with strong rotational forces and clearance between the rotating impeller and stationary shroud generate secondary flows which, in unshrouded impellers, transport low-momentum fluid into the mainstream of the impeller passage. The ability to reduce the resultant flow nonuniformities at the impeller exit by altering new impeller designs to control the secondary flow development may therefore lead to improved performance. In order for such a design activity to succeed, it must be guided by an improved understanding of the flow physics within the impeller and by reliable numerical flow field predictions.

Several previous investigators provided flow field measurements within unshrouded centrifugal compressor impellers. Eckardt's laser anemometer measurements in a radial outflow impeller provided the first experimental evidence in high-speed impellers of the development of a "wake" of low-momentum fluid near the suction surface/ shroud corner of the blade passage (Eckardt, 1976). Krain (1988), Krain and Hoffman (1990), Ahmed and Elder (1990), Sipos (1991), and Rohne and Banzhaf (1990) acquired laser anemometer measurements in backswept impellers which indicate that the wake develops near the shroud at midpitch.

As a result of the small passage size and optical access limitations in these previous investigations, few experimental details of the secondary flow development inside high-speed impeller passages are available. Krain (1988) and Sipos (1991) therefore used vortex models to infer the vortical nature of the secondary flow from the flow angle measurements which they were able to acquire on blade-to-blade stream surfaces.

Several low-speed investigations also provided some insight into secondary flows. Senoo, Yamaguchi, and Nishi (1968) used dye in a water-flow experiment to visualize the tip clearance flow. Farge, Johnson, and Maksoud (1989) performed five-hole pressure measurements in a 1-m-diameter shrouded impeller which rotated at $500 \mathrm{rpm}$. A clearance gap was left between the blade tip and the impeller shroud in order to generate a tip clearance flow. However, as the authors point out, there is no relative motion between the blade and the shroud so the physics of the tip clearance flow in this investigation is not the same as it would be in an unshrouded impeller. Fagan and Fleeter (1991) used laser anemometry to measure all three velocity components in a low-speed, shrouded, mixed-flow compressor.

In lieu of detailed experimental measurements of the secondary flow fields in unshrouded impellers, several investigators used three-dimensional Navier-Stokes numerical solutions to generate secondary flow details (Krain and Hoffman, 1989, 1990; Hah and Krain, 1990; Bansod and Rhie, 1990; Moore and Moore, 1990). Fagan and Fleeter (1991) also presented calculated secondary flow field results for their shrouded impeller.

The purpose of the present investigation is to provide a detailed experimental study of primary and secondary flow development within an unshrouded centrifugal compressor impeller. A low-speed centrifugal compressor (LSCC) which has an exit diameter of $1.52 \mathrm{~m}$ was specifically designed and commissioned to meet this objective. The LSCC was designed to generate a flow field that is aerodynamically similar to that found in high-speed subsonic centrifugal compressors. The large size of the impeller enables the 
measurement of all three velocity components throughout the impeller blade passage.

Laser anemometer measurements and surface flow visualization results are presented and compared with a prediction of the flow field generated using Dawes' three-dimensional Navier-Stokes code (Dawes, 1988). The processes which lead to the formation of the throughflow momentum wake that is characteristic of unshrouded centrifugal compressor impellers are clearly explained using the results from both CFD and experiment. The results indicate that low-momentum fluid near the blade surfaces migrates outward toward the tip of the blade and becomes entrained in the tip clearance jet. In addition, low-momentum fluid near the blade tip is transported to the pressure side/shroud corner of the blade passage where it accumulates to form the wake.

Measurements acquired downstream of the impeller are also presented to illustrate how the flow field nonuniformities existing at the impeller exit mix out through the vaneless diffuser. Finally, the flow field measurements obtained in the LSCC are compared with those measured in high-speed impellers by Krain and Hoffman $(1989,1990)$ to assess the degree to which the flow physics in the LSCC simulates the flow physics within a high-speed centrifugal impeller.

\section{Symbols}

unit vector in measured velocity component direction

$\vec{g} \quad$ local streamwise grid direction vector

$\hat{g}_{m} \quad$ unit vector in local meridional grid direction

$\hat{g}_{p} \quad$ unit vector in local pitchwise grid direction

$\hat{g}_{s} \quad$ unit vector in local spanwise grid direction

J streamwise measurement grid index

$\mathrm{m} / \mathrm{m}_{\mathrm{s}}$ nondimensional shroud meridional distance

$N_{c p p} \quad$ number of encoder counts per blade pitch

$N_{p} \quad$ number of impeller blade passages

PS pressure surface

$r / r_{t} \quad$ radius nondimensionalized by exit tip radius

SS suction surface

$U_{t} \quad$ impeller tip speed, $\mathrm{m} / \mathrm{sec}$

$\vec{V} \quad$ absolute total velocity vector, $\mathrm{m} / \mathrm{sec}$
$V_{c} \quad$ velocity component measured in direction $\hat{c}, \mathrm{~m} / \mathrm{sec}$

$\vec{V}_{g} \quad$ projection of $\vec{V}_{T}$ in local streamwise direction $\vec{g}$

$V_{m} \quad$ meridional velocity component, $\mathrm{m} / \mathrm{sec}$

$V_{p} \quad$ pitchwise secondary velocity component, $\mathrm{m} / \mathrm{sec}$

$V_{q m} \quad$ quasi-meridional velocity component, $\mathrm{m} / \mathrm{sec}$

$V_{r} \quad$ radial velocity component, $\mathrm{m} / \mathrm{sec}$

$V_{s} \quad$ spanwise secondary velocity component, positive towards the shroud, $\mathrm{m} / \mathrm{sec}$

$V_{s t} \quad$ velocity component tangent to the shroud meridional direction, $\mathrm{m} / \mathrm{sec}$

$\vec{V}_{T} \quad$ total relative velocity, $\mathrm{m} / \mathrm{sec}$

$V_{z} \quad$ axial velocity component, $\mathrm{m} / \mathrm{sec}$

$\mathrm{V}_{\theta} \quad$ relative tangential velocity component, $\mathrm{m} / \mathrm{sec}$

$\alpha \quad$ flow pitch angle, deg, $\alpha=\tan ^{-1}\left(V_{r} / V_{z}\right)$

$\beta \quad$ absolute flow angle, deg, $\beta=\tan ^{-1}\left(V_{\theta} / V_{q m}\right)$

$\theta \quad$ tangential coordinate, rad

Superscript:

- $\quad$ passage average

\section{Test Compressor}

The NASA low-speed centrifugal compressor (LSCC) is an experimental facility designed to duplicate the essential flow physics of high-speed subsonic centrifugal compressor flow fields in a large low-speed machine in which very detailed investigations of the flow field can be made. A complete description of the facility is provided by Wood, Adam, and Buggele (1983) and Hathaway, Wood, and Wasserbauer (1992).

The test compressor is a backswept impeller with a design tip speed of $153 \mathrm{~m} / \mathrm{sec}$. The impeller is followed by a vaneless diffuser in order to generate an axisymmetric outflow boundary condition which is desirable for a CFD analysis of an isolated blade row. The impeller has 20 full blades with a backsweep of $55^{\circ}$. The inlet diameter is $0.870 \mathrm{~m}$ and the inlet blade height is $0.218 \mathrm{~m}$. The exit diameter is $1.524 \mathrm{~m}$ and the exit blade height is $0.141 \mathrm{~m}$. The tip clearance between the impeller blade and the shroud is $2.54 \mathrm{~mm}$ and is constant from the impeller inlet to the impeller exit. This tip clearance is 1.8 percent of the blade height at 
the exit of the impeller. The blade surfaces are composed of straight-line elements from hub to tip, a feature that enabled the fabrication of the impeller by a flank-milling process on a five-axis milling machine. This feature also facilitates the acquisition of laser anemometer measurements of velocities close to the blade surfaces because the laser anemometer optical axis can be directed parallel to the blade surface.

The research operating point selected for both computational and experimental investigations of the LSCC flow field was set at standard-day-corrected conditions of $30 \mathrm{~kg} / \mathrm{sec}$ and $1862 \mathrm{rpm}$, which is near peak efficiency, for all data presented herein.

\section{Instrumentation and Measurement}

\section{Technique}

Separate laser windows provide optical access to the impeller in each of three regions: the impeller inlet, knee, and exit. Each window has compound curvature and matches the shroud contour in both the streamwise and circumferential directions to maintain the proper impeller tip clearance throughout the compressor. A meridional view of the LSCC (fig. 1) shows the spanwise and streamwise locations at which laser anemometer data were acquired. The cross-channel measurement stations are numbered to denote the stations at which data are presented. The station numbers are the streamwise indices of a body-fitted measurement grid used to position the laser measurement point in the impeller. The measurement grid used in this investigation divides the

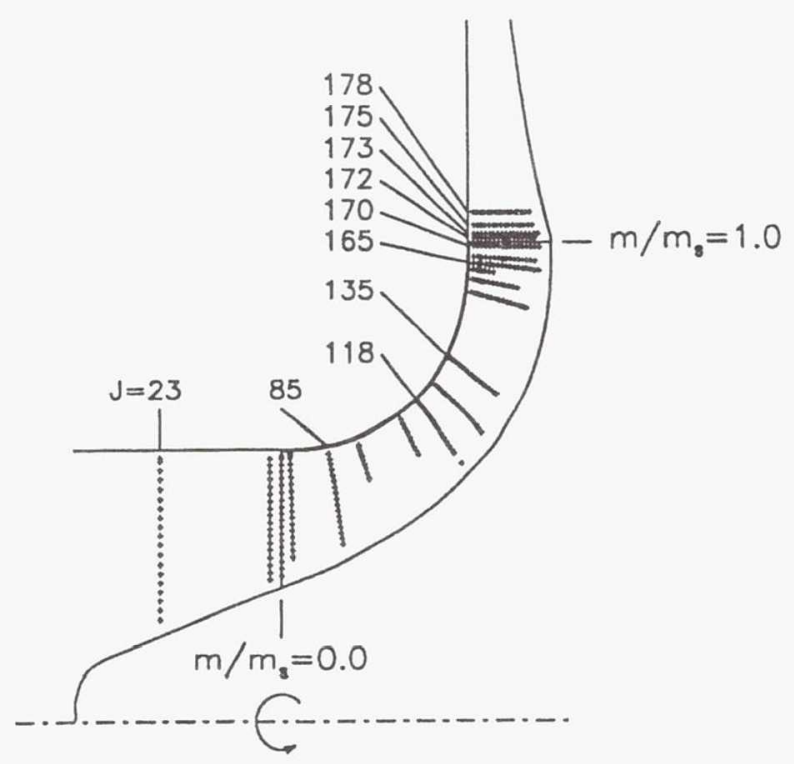

Figure 1.-Meridional view of low-speed centrifugal compressor (LSCC) rotor showing laser anemometer measurement locations. Nondimensional shroud meridional distance, $\mathrm{m} / \mathrm{m}_{\mathrm{s}}$. streamwise blade length into a series of quasi-orthogonal, or near-normal, cross-channel planes. Stations $23,85,118,135$, 165 , and 170 are located at $-40,14,47,64,94$, and 99 percent of the meridional shroud length. The four measurement stations in the vaneless diffuser $(172,173,175$, and 178) are at impeller tip radius ratios of $1.010,1.019,1.037$, and 1.065 .

\section{Laser Anemometer and Data Acquisition}

A two-component laser fringe anemometer operating in an on-axis backscatter mode was used in this investigation. Frequency shifting was employed for both fringe systems to provide directional sensitivity for all velocity measurements.

Two digital shaft angle encoders (one for each laser anemometer channel) were used to generate a fixed number of pulses for each impeller revolution. When a laser velocity measurement occurred, the current encoder count was recorded along with the velocity data. The encoder count indicates the circumferential location of the velocity measurement relative to a once-per-revolution timing mark on the impeller. In the present investigation, the encoders were set to generate the number of encoder counts per blade pitch $N_{c p p}$ at 200 across each of the 20 impeller blade channels. Further details of the encoding technique are described by Strazisar et al. (1989) and Wood, Strazisar, and Hathaway (1990).

The velocity component measurements acquired at each encoder count are first arithmetically averaged to determine the mean of each measured velocity component $V_{c}$ where subscript $c$ denotes that the velocity was measured in the direction of the unit vector. The mean velocities are then averaged across the 20 blade channels as shown in equation (1) to yield a single "passage-averaged" velocity profile for each velocity component that is representative of the profile across each individual blade passage:

$$
\bar{V}_{c}(i)=\frac{1}{N_{p}} \sum_{j=1}^{N_{p}} V_{c}\left(i+N_{c p p} *(j-1)\right), i=1, N_{c p p}
$$

The normalized difference is

$$
\frac{\bar{V}_{c}(i)-V_{c}\left(i+N_{c p p} *(j-1)\right)}{\bar{V}_{c}(i)}
$$

where $\bar{V}_{c}(i)$ is the passage-averaged velocity component at any pitchwise location $i$, and $V_{c}\left(i+N_{c p p} *(j-1)\right)$ is the mean velocity component in blade passage $j$ at the same pitchwise location. The normalized difference was checked and was generally less than 1 percent, which indicates that the passage-averaging process did not appreciably alter any of the blade-to-blade velocity profile details. All the data presented in this report are based on passage-averaged velocity distributions. 
In most regions of the flow field, 50000 velocity measurements were acquired at each (axial, radial) measurement point shown in figure 1 . For measurements evenly distributed across the blade pitch, there would be an average of 250 measurements at each of the 200 points in the passage-averaged distribution. However, the number of measurements was generally not evenly distributed across the pitch. Therefore, the total number had to be adjusted to maintain a reasonable number of measurements in regions across the pitch where the data rate was low.

\section{Seeding Technique}

Polystyrene latex (PSL) spheres, manufactured according to the process developed by Nichols (1987), were used as the seed material. Because of limitations in the manufacturing tolerance, the mean size of the seed particles produced for a given batch of seed varied from batch to batch although the standard deviation of the particle sizes for a given batch of seed was generally less than 0.01 to $0.03 \mu \mathrm{m}$. The mean size of the seed particles used during the course of this investigation varied from 0.8 to $0.95 \mu \mathrm{m}$.

The seed material manufactured by Nichols' technique consists of a dilute suspension of PSL spheres in water. This mixture is further diluted in pure ethyl alcohol. Air-driven atomizing nozzles are used to inject the seed-water-alcohol mixture into the airstream approximately $3 \mathrm{~m}$ upstream of the impeller leading edge. The alcohol and water quickly evaporate, leaving individual PSL particles suspended in the airstream.

Prior to making laser anemometer measurements, extensive in-situ particle size measurements were made with an aerodynamic particle sizer to insure that the seeding scheme was operating properly (Wasserbauer and Hathaway, 1993). The sizer was used to analyze airstream samples which were extracted through a pitot tube located at measurement station 23 ahead of the impeller. No particles could be detected at this location when pure alcohol was injected through the spray nozzles. The sizer was also used to analyze air samples when seed particles were added to the alcohol carrier to insure that individual seed particles did not agglomerate into groups of larger particles.

\section{Three-Dimensional Velocity Measurement Technique}

To determine all three components of the total velocity vector at a point in the flow field, two velocity components are measured at each of two different orientations of the laser anemometer optical axis. The two orientations of the optical axis were selected to minimize the amount of the blade passage which is optically blocked by the blade. At the same time, a $20^{\circ}$ to $30^{\circ}$ included angle between the two orientations is maintained to minimize the propagation of the measured velocity component uncertainty in the calculated components. The resultant four measured components are combined using a least-squares fit (eq. (3)) to yield the total three-dimensional velocity vector

$$
\frac{\partial\left\{\sum_{k=1}^{4}\left[\vec{V} \cdot \hat{c}_{k}-\left(\bar{V}_{c}\right)_{k}\right]^{2}\right\}}{\partial \vec{V}}=0
$$

where $\vec{V}$ is the absolute total velocity vector to be determined, $\hat{c}_{k}$ is the unit vector in the $k^{\text {th }}$ measurement direction, and $\left(\bar{V}_{c}\right)_{k}$ is the measured passage-averaged velocity component in the direction of $\hat{c}_{k}$. As a check, the projection of the calculated total vector in the direction of each of the four measured components is compared with the measured component. The difference is generally less than 2 percent of the measured component.

The emphasis in the measurement technique just described was to obtain the spanwise velocity component because this component was not measured by most previous laser anemometer investigations in centrifugal compressors and is

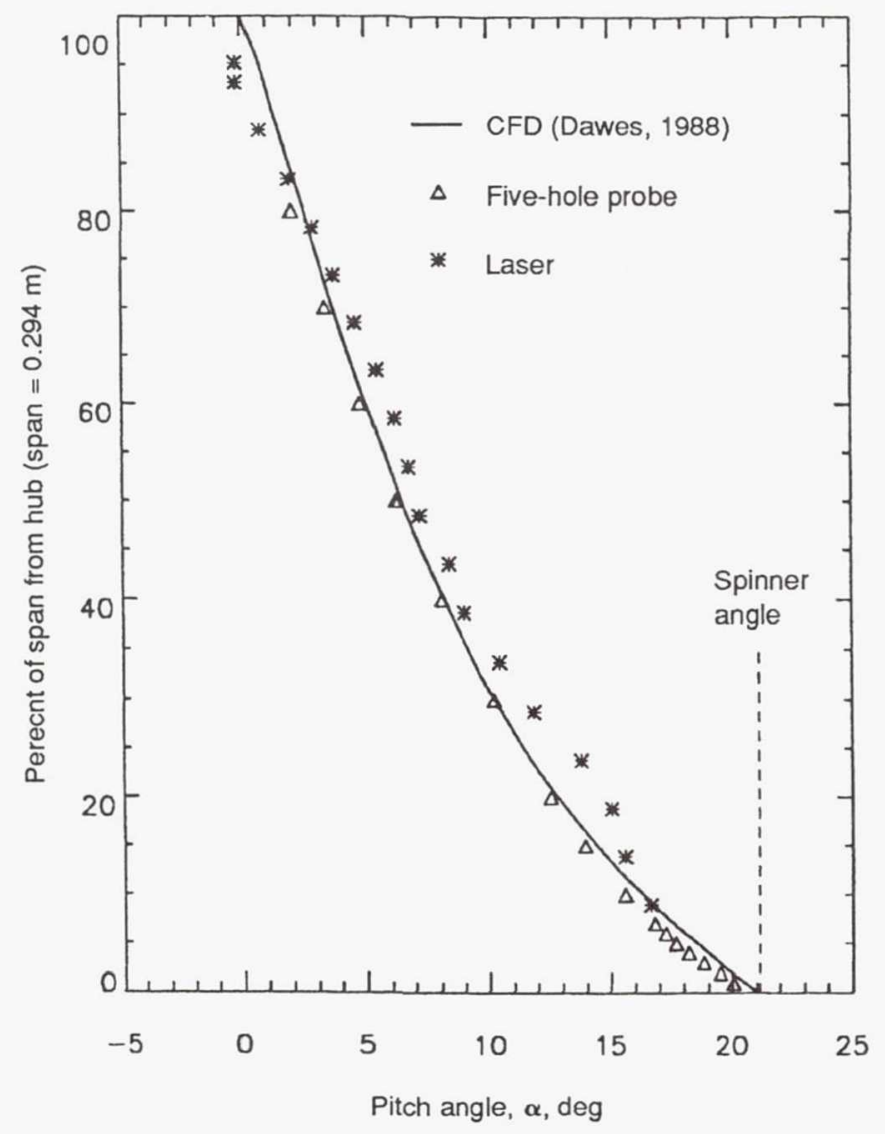

Figure 2.-Measured and predicted pitch angle distribution at rotor inlet station $\mathrm{J}=23$. 
by far the most difficult component to resolve in most turbomachinery applications. The ability to measure the spanwise velocity component was verified upstream of the impeller at station 23 (shown in fig. 1) by comparing the pitch angle $\alpha$, derived from the laser anemometer measurements, with the pitch angle measured by a five-hole probe. The results of this exercise (fig. 2) indicate that the laser measurement technique is capable of accurately measuring the relatively small pitch angles that exist near the shroud. The agreement between the laser and five-hole probe measurements of the pitch angle is better than $2^{\circ}$ over the outer 70 percent of span ( $0.20-\mathrm{m}$ immersion). The laser measurements tend to depart from the five-hole probe measurements at lower spans, probably as a result of the effect of the window curvature which distorts the laser measurement volume.

\section{Measurement Uncertainty}

The uncertainty of the individual velocity component measurements was estimated from the least-squares calculation to be on the average approximately $1.5 \mathrm{~m} / \mathrm{sec}$ throughout most of the impeller passage. Through much of the impeller passage, the throughflow velocity magnitude is on the order of $75 \mathrm{~m} / \mathrm{sec}$. Thus, the uncertainty of the measured velocity components is less than 2 percent of the throughflow component.

In addition to the propagation of the measured velocity component uncertainties in the calculated velocity components, the measured velocity components are subject to the uncertainties arising from window curvature effects that distort the laser anemometer probe volume. The spanwise velocity component and flow pitch angle are most susceptible to uncertainty propagation and are therefore most sensitive to window curvature effects. Based on the results shown in figure 2 , the uncertainty in pitch angle, which directly indicates the ability to resolve the spanwise velocity component, is estimated to be less than $2^{\circ}$ for measurement locations in the outer 70 percent of the blade span for all measurement stations up to and including station 135. Because window curvature and blade span both decrease in the rear of the impeller, the uncertainty in the pitch angle for measurement stations 165 to 178 should be less than $2^{\circ}$ over the entire blade span.

\section{Computational Analysis}

The computational results for the LSCC flow field were obtained using the Reynolds-averaged Navier-Stokes code developed by Dawes (1988). The code solves the equations of motion in cylindrical coordinates in integral conservation form using six-sided control volumes formed by a simple H-mesh. The basic algorithm as described by Dawes (1988) is similar to a two-step Runge-Kutta method plus residual smoothing. A combined second- and fourth-derivative artificial viscosity model with pressure gradient switching was used to eliminate spurious "wiggles" and to control shock capturing. The eddy viscosity was obtained using the Baldwin-Lomax (1978) mixing length model. Tip clearance was handled by gradually decreasing the thickness of the blade to zero in the tip clearance and enforcing periodicity. Because this simple treatment of the tip clearance does not solve for the details in the tip clearance gap, the actual tip clearance used in the code was reduced to 60 percent of the physical tip clearance to simulate the "vena-contracta" formed by the tip clearance jet.

The solution grid has 137 streamwise points ( 75 points are within the blade) with clustering around the leading edge, 71 points spanwise with 4 volumes in the tip clearance, and 41 points pitchwise. The pitchwise spacing at midpitch is about 5 percent of pitch, and the nearest points away from the blade surfaces are at about 1 percent of pitch (however, the cell centers are 0.5 percent of pitch from the surfaces). The spanwise spacing varies from a maximum of about 5 percent of span at midspan to 0.15 percent of span for the grid nodes nearest the hub and shroud (the cell centers are about 0.07 percent away from the endwalls). The maximum streamwise spacing is about 2.5 percent of meridional chord at midchord.

The actual spinner geometry was modeled by fitting a small-diameter cylindrical section to the spinner at a radius of $107 \mathrm{~mm}$. In the calculation the spinner rotation was started at grid line 8 , which is 54 percent of the shroud meridional chord upstream of the blade leading edge and about 12 percent of the chord upstream of measurement station 23 . The impeller hub rotation was stopped just downstream of the trailing edge.

Convergence was determined when the mass flow error throughout the domain was deemed acceptable and the losses through the compressor stabilized. Also, velocity components at selected points in the grid were monitored to insure that the velocity field was not changing. The maximum mass flow error was 0.56 percent of the inlet flow and occurred near the downstream boundary. The maximum mass flow error in the compressor blade passages was 0.38 percent and occurred near the trailing edge.

\section{Results and Discussion}

Computational fluid dynamics analysis has been used in a synergistic fashion with the experimental effort throughout the course of the LSCC research program and this investigation is no exception. Prior to the experiment, a coarse-grid flow field prediction was first generated using Dawes' three-dimensional Navier-Stokes code (Hathaway, Wood, and Wasserbauer, 1992) to develop an understanding of the flow 
field characteristics and to aid in planning the location of laser anemometer measurements. The final CFD solution, presented herein, was used to further our understanding of flow physics phenomena (such as the tip clearance flow) which were not completely captured during the experimental effort.

The results presented consist of laser anemometer measurements of the velocity field along several quasi-orthogonal planes through the impeller. Ammonia-ozalid surface flow visualization results within the impeller and hot-wire anemometer results obtained in the vaneless diffuser are also presented. The main objective of the data and computational analysis is to describe the complex flow physics within a subsonic centrifugal compressor and to demonstrate the fidelity of the three-dimensional Navier-Stokes flow field analysis.

The laser anemometer and CFD results which document the development of the throughflow velocity distribution through the impeller are presented first. Secondary velocity vector plots are then presented to illustrate the migration of low-momentum fluid through the impeller blade passage. Blade-to-blade distributions of the throughflow and secondary velocity components and flow pitch angles are also presented at a few points of interest within the impeller. Finally, pitchwise distributions of the absolute flow angle are presented to illustrate the mixing of the impeller exit flow in the vaneless diffuser.

\section{Throughflow Development}

The streamwise development of the throughflow velocity is illustrated through the use of "wire-frame" plots of the quasi-meridional velocity normalized by the impeller tip speed $V_{q m} / U_{t}$. As described in the appendix, the quasimeridional velocity $V_{q m}$ approximates the throughflow velocity component at any cross-channel station in the impeller. In order to directly compare the CFD solution to the laser data, the CFD solution was first interpolated in the streamwise direction to obtain predicted values of the velocity components $V_{r}, V_{\theta}, V_{z}$ at the experimental measurement planes shown in figure 1 . On each plane, the CFD solution was then interpolated to the same spanwise locations as the laser data. The velocity components $V_{q m}, V_{s}, V_{p}$, defined in the appendix, were then determined in the same manner for both experiment and CFD relative to the experimental measurement grid. There is therefore a one-to-one correspondence between the blade-to-blade velocity profiles in the CFD and experimental wire-frame plots. The results in each plot are shown at every 5 percent of span, with the results nearest the shroud located at 95 percent of blade span from the hub (where 100 percent of span denotes the blade tip).

The complex nature of the flow field can lead to concern over the impact of interpolating the CFD results to the laser measurement locations. Therefore, for each measurement station presented in this paper, we compared the CFD results on the two quasi-orthogonal CFD calculation planes which lie on either side of the laser measurement plane to the CFD results which were interpolated to the measurement plane. In all cases we found that the interpolation process did not alter the predicted flow field characteristics.

Favorable agreement between CFD predictions and the laser anemometer measurements was obtained at station 85 (nondimensional shroud meridional distance $\mathrm{m} / \mathrm{m}_{s}=0.149$ ), as shown in figure 3(a). Both the CFD and experimental results indicate an almost linear velocity distribution across the blade passage. The results obtained at station $118\left(\mathrm{~m} / \mathrm{m}_{s}=0.475\right)$ are shown in figure $3(\mathrm{~b})$. At this station, a region of low streamwise momentum appears near the shroud at about midpitch. This region, referred to as the wake region by previous investigators (Eckardt, 1976; Krain, 1988), is evident in the experimental data at both 90 and 95 percent of span but is only evident in the CFD solution at 95 percent of span. An inspection of data which are not presented here indicates that the wake first begins to develop at midpitch at $\mathrm{m} / \mathrm{m}_{s}=0.40$.

The results obtained at station $135\left(\mathrm{~m} / \mathrm{m}_{s}=0.644\right)$ are shown in figure $3(\mathrm{c})$. The broken lines indicate additional results obtained at intervals of 1 percent of span from 96 to 100 percent of span. The momentum deficit near the shroud in both the measurements and the CFD results continues to increase in magnitude. The wake centerline also moves toward the pressure side of the passage. The measured wake occupies the outer 25 percent of the span. Note that the CFD solution underpredicts both the maximum velocity deficit in the wake and the spanwise extent of the wake. Also note from figures 3(a) to (c) that both the measured and predicted throughflow momentum in the portion of the passage which is outside the wake are nearly constant from stations 85 to 135 .

At station $165\left(\mathrm{~m} / \mathrm{m}_{s}=0.941\right)$ shown in figure 3(d), the measured throughflow velocity deficit in the wake is less severe than it was at station 135 . However, the minimum wake velocity at 95 percent of span is nearly unchanged between stations 135 and 165. A detailed inspection of the blade-to-blade distributions of the throughflow velocity, plotted at individual spanwise locations, indicates that the streamwise velocity throughout the lower half of the passage and near the suction surface in the upper half of the passage has dropped significantly relative to its level at station 135 . This behavior makes the wake appear less severe at station 165 than at station 135 . The CFD solution indicates the wake has grown to occupy a larger spanwise extent than at station 135 and that the predicted minimum wake velocity is now comparable to the measured wake minimum velocity. Station 165 is in the region of the impeller blade passage where the pressure surface is not "covered" by the suction surface. This region begins at $m / m_{s}=0.85$. The observed increase in pressure surface velocity and decrease in suction surface velocity is expected as a result of blade unloading. There is also a shift of the wake centerline toward midpitch.

The results obtained at station $170\left(\mathrm{~m} / \mathrm{m}_{\mathrm{s}}=0.990\right)$ are shown in figure 3(e). The CFD results agree qualitatively 

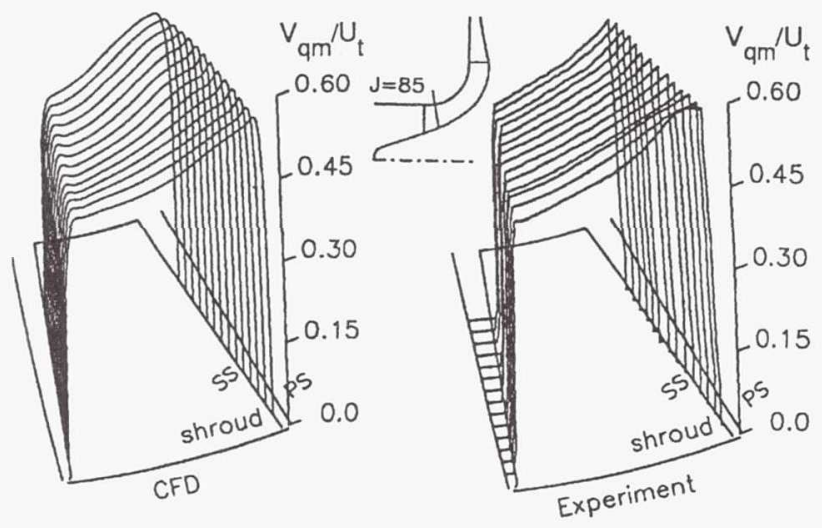

(a) Station $85\left(\mathrm{~m} / \mathrm{m}_{\mathrm{s}}=0.149\right)$.

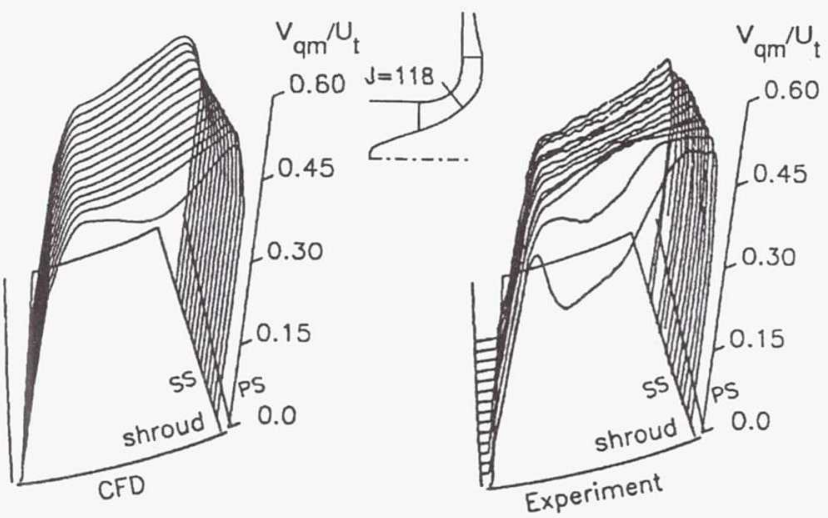

(b) Station $118\left(\mathrm{~m} / \mathrm{m}_{\mathrm{s}}=0.475\right)$.

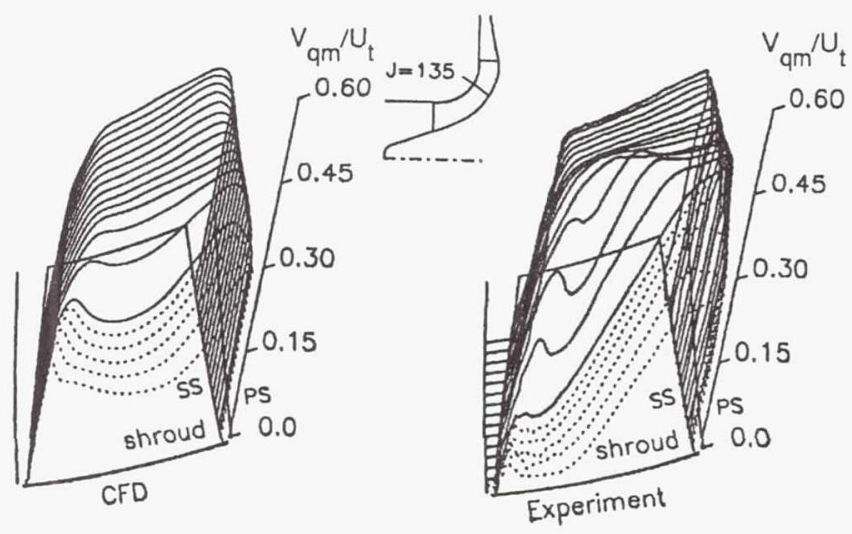

(c) Station $135\left(\mathrm{~m} / \mathrm{m}_{\mathrm{s}}=0.644\right)$.

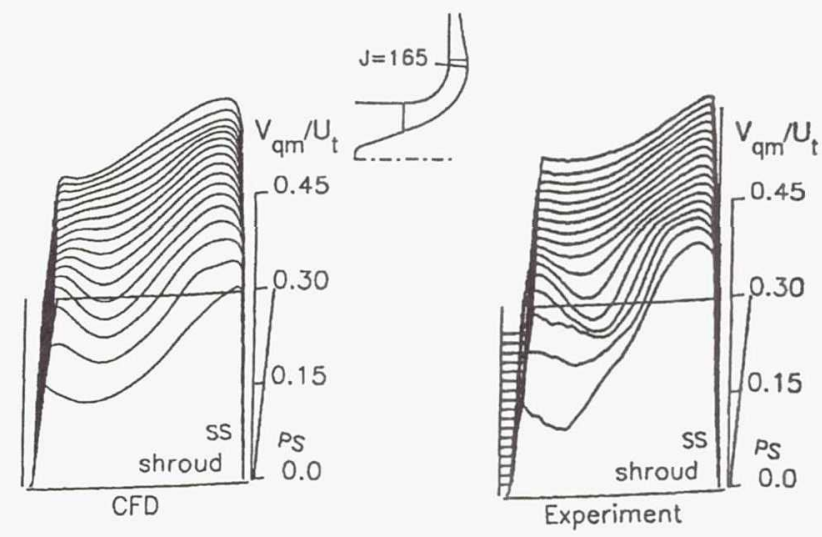

(d) Station $165\left(\mathrm{~m} / \mathrm{m}_{\mathrm{s}}=0.941\right)$.
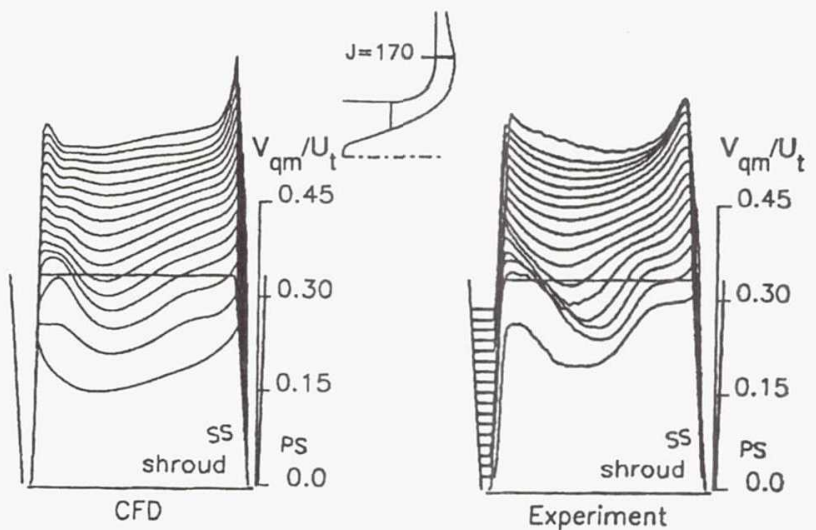

(e) Station $170\left(\mathrm{~m} / \mathrm{m}_{\mathrm{s}}=0.990\right)$.

Figure 3.-Distribution of quasi-meridional velocity nondimensionalized by impeller tip speed $\mathrm{V}_{q m} / U_{t}$ at five stations at intervals of 5 percent of span starting at 95 percent of span.

with the measurements. As we move from station 165 to station 170 , we also note a continued unloading of the blade as well as a continued shift of the wake centerline toward midpitch. The shift in wake centerline location near the exit of the impeller results from a continuing increase in the throughflow velocity near the pressure surface with increasing downstream distance. This behavior can be seen more clearly in figure 4 where the measured blade-to-blade distributions of throughflow velocity at 90 percent of span for stations 135 to 170 are plotted. 


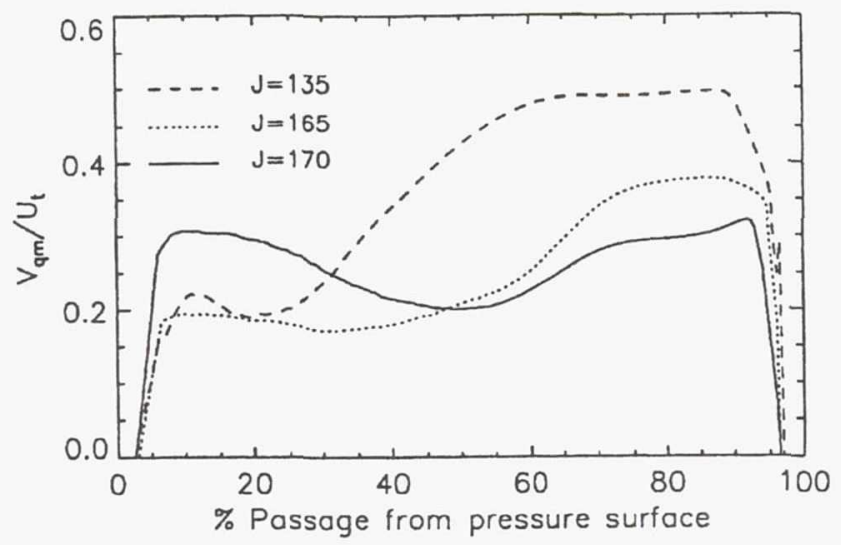

Figure 4.-Blade-to-blade distribution of measured quasimeridional velocity at 90 percent of span for several stations near exit of impeller.

In summary, the results shown in figure 3 indicate that the classic throughflow momentum deficit, or wake, which has been observed in centrifugal compressors by many other investigators occurs in the outer 25 to 35 percent of the span at the blade exit for this compressor. The wake is initially detected near midpitch, moves to the pressure surface/shroud corner of the passage, and then moves toward midpitch because of a continued increase in momentum in the pressure surface/shroud corner of the blade passage near the exit of the impeller.

The CFD solution predicts the location and strength of the wake near the impeller exit quite well but underpredicts the maximum velocity deficit in the wake and the spanwise extent of the wake at stations 118 and 135 . These features of the CFD solution are quite sensitive to the tip clearance model used in the code. The code models the vena-contracta, formed by the clearance jet crossing the sharp blade edge, by using a tip clearance value which is less than the physical tip clearance. The value used in the present solution is 60 percent of the physical tip clearance. The effect of increasing the tip clearance used in the code from 60 to 100 percent of the actual clearance has been studied using a slightly coarser grid ( 75 streamwise points in the blade, 41 points spanwise, 41 points pitchwise). The results indicate that increasing the tip clearance in the CFD solution does indeed result in an increase in the spanwise extent of the wake and moves the wake minimum velocity closer to the pressure surface at a given station. Inspection of the impeller blade tips at the conclusion of testing indicated that the blade tip corners were not sharp. A contraction coefficient of 0.6 is therefore too small. Calculations using a larger contraction coefficient will be pursued in a future analysis of this impeller.

\section{Secondary Velocity Development}

The objective of this section is to illustrate how the secondary velocity field within the impeller contributes to the formation and movement of the throughflow wake within the impeller. The development of the "secondary velocity" field will be documented by presenting secondary velocity vector plots on the cross-channel measurement planes. Secondary velocity as used in this paper is defined as that component of the total relative velocity vector which is not aligned with streamwise CFD grid lines. A detailed definition of the spanwise and pitchwise secondary velocity components is given in the appendix. Measured and predicted secondary velocity vectors are calculated in the same way.

As in the previous section, the CFD results were interpolated to the streamwise and spanwise location of the laser measurements. To provide more details of the tip clearance flow, CFD results are also shown at both 98 and 101 percent of blade span, which lies inside the tip clearance gap. The pitchwise location of each vector in the CFD results is fixed by the pitchwise location of the CFD grid nodes. Although the laser measurements were made at 200 points across the pitch, only the data at every third point are presented for the sake of clarity. The predicted and measured vectors are drawn to the same scale with a reference vector of length $0.5 U_{t}$ shown in each plot. The streamwise momentum outside the wake region for measurement stations up to and including station 135 is on the order of $0.5 U_{t}$. The strength of the secondary flow relative to the throughflow can therefore be approximated by comparing the secondary velocity vector magnitudes to the reference vector magnitude in each plot.

Also shown in the predicted secondary velocity vector plots are the locations of CFD tracer particles which were released along the blade leading edge from hub to tip on either side of the stagnation line. The CFD tracer locations illustrate how low-momentum fluid along the blade surface migrates toward the blade tip and becomes entrained in the tip clearance flow.

The secondary flow at station 85 near the impeller inlet is shown in figure 5(a). The CFD result shows a spanwise flow of fluid along the pressure surface in the outer 50 percent of the blade span. A fairly weak vortical flow is predicted at this station and the experiment is just beginning to indicate some vortical flow near the shroud. Some of the CFD tracer particles released on the suction surface at the leading edge, denoted by asterisks, have already migrated to the tip of the blade, have been entrained in the tip clearance jet, and have begun to move toward the pressure side of the passage. Some of the CFD tracer particles released on the pressure surface at the leading edge, denoted by diamonds, have migrated to the tip of the blade, have crossed over the tip, and have also begun to move toward the pressure side of the passage.

At station 118 shown in figure 5(b), both the CFD and experimental results indicate that the low-momentum fluid near the blade suction surface is migrating outward toward the blade tip. The CFD solution also indicates a continuing migration of fluid toward the tip along the pressure surface. However, the experimental results indicate that the fluid 
CFD

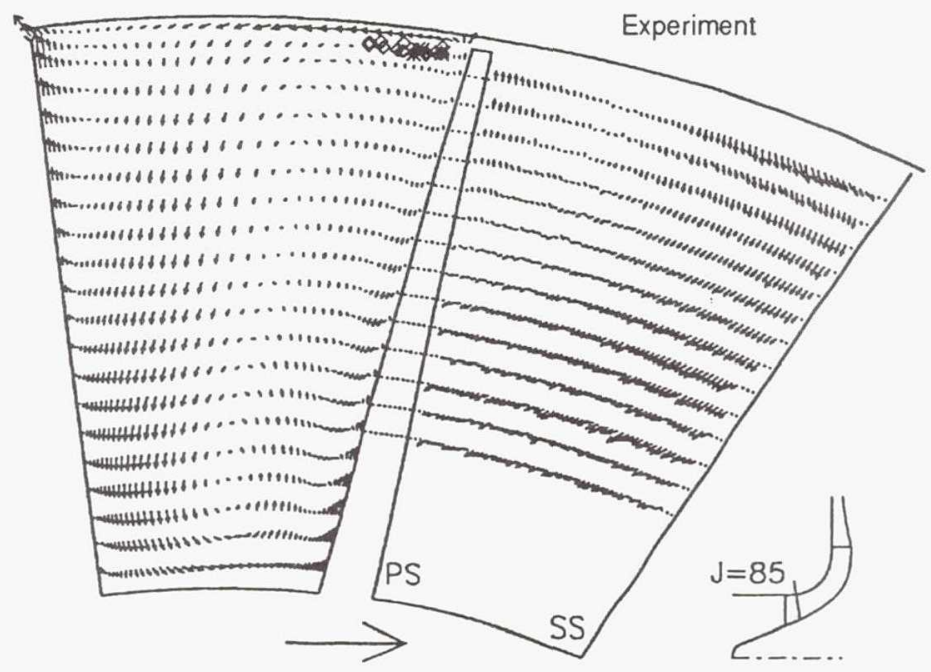

$0.5 U_{\mathrm{t}}$

(a) Station $85\left(\mathrm{~m} / \mathrm{m}_{\mathrm{s}}=0.149\right)$.

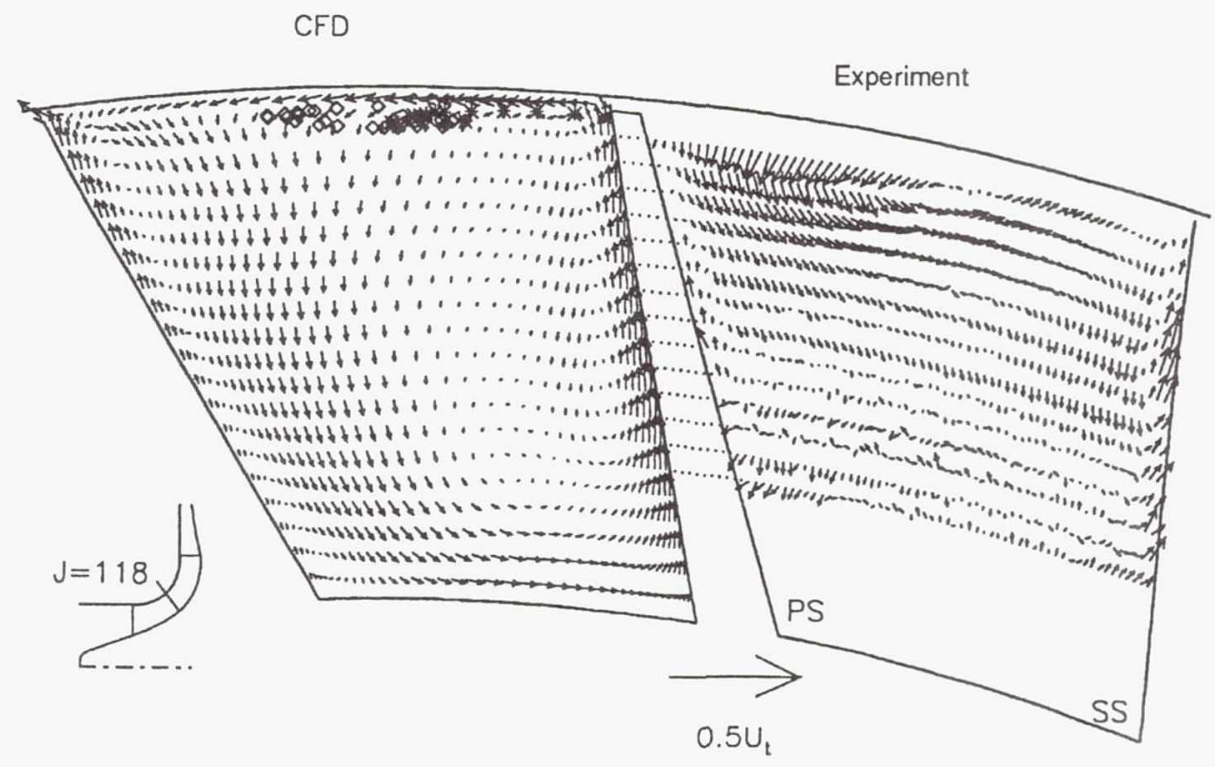

(b) Station $118\left(\mathrm{~m} / \mathrm{m}_{\mathrm{s}}=0.475\right)$.

Figure 5.-Secondary flow velocity vector plots at five stations. Both CFD and experimental results shown at intervals of 5 percent of span starting at 95 percent of span with additional CFD results at 98 and 101 percent of span. Diamonds and asterisks denote CFD tracers released along blade leading edge on pressure and suction surfaces, respectively.

moving outward along the blade pressure surface is met and turned by fluid from the vortical flow near the shroud which is moving inward toward the hub near the pressure surface/ shroud corner. The vortical flow near the shroud resides in approximately the same location as the low-momentum throughflow wake region indicated in figure 3 (b). The CFD and experimental results do not provide a clear indication of the origin of the inward flow which is measured in the pressure surface/shroud corner. However, the CFD tracer locations indicate that the blade leading edge particles that are now entrained within the tip clearance flow are migrating toward the pressure surface. The inward flow in the blade 

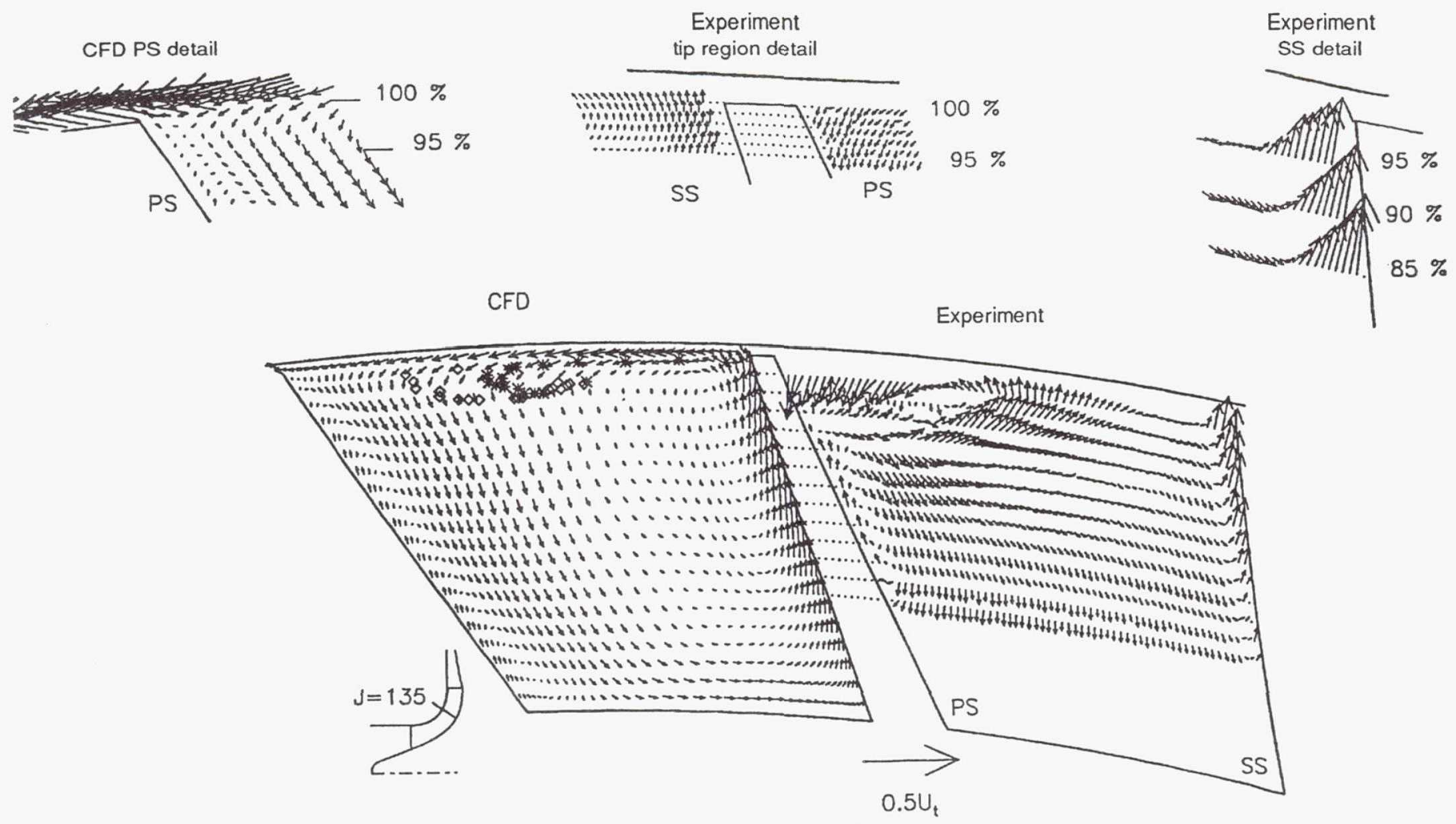

(c) Station $135\left(\mathrm{~m} / \mathrm{m}_{\mathrm{s}}=0.644\right)$.

Secondary velocity vectors based on mode

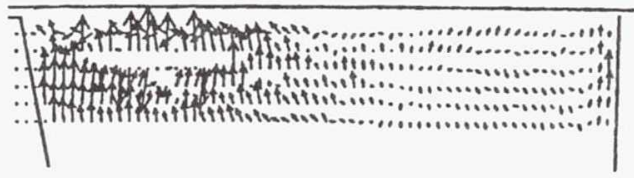

CFD

Experiment

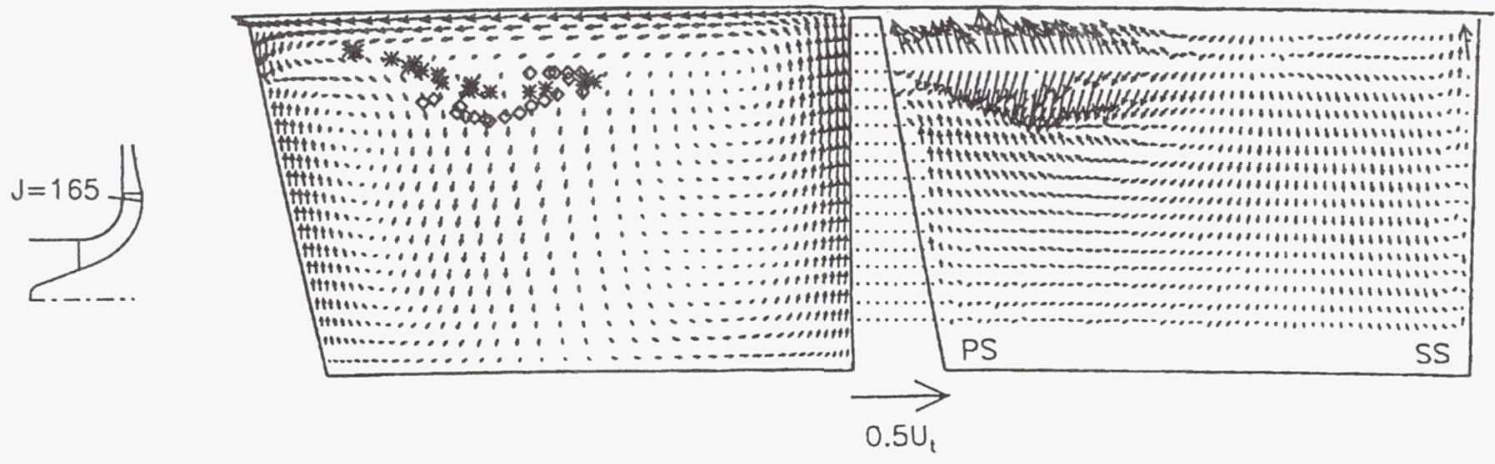

(d) Station $165\left(\mathrm{~m} / \mathrm{m}_{\mathrm{s}}=0.941\right)$.

Figure 5.-Continued.

pressure surface/shroud corner may therefore be a result of the tip clearance flow impinging on the blade pressure surface.
The secondary flow results obtained at station 135 are shown in figure $5(\mathrm{c})$. Both the CFD and experimental results indicate a continuing migration of fluid outward toward the 


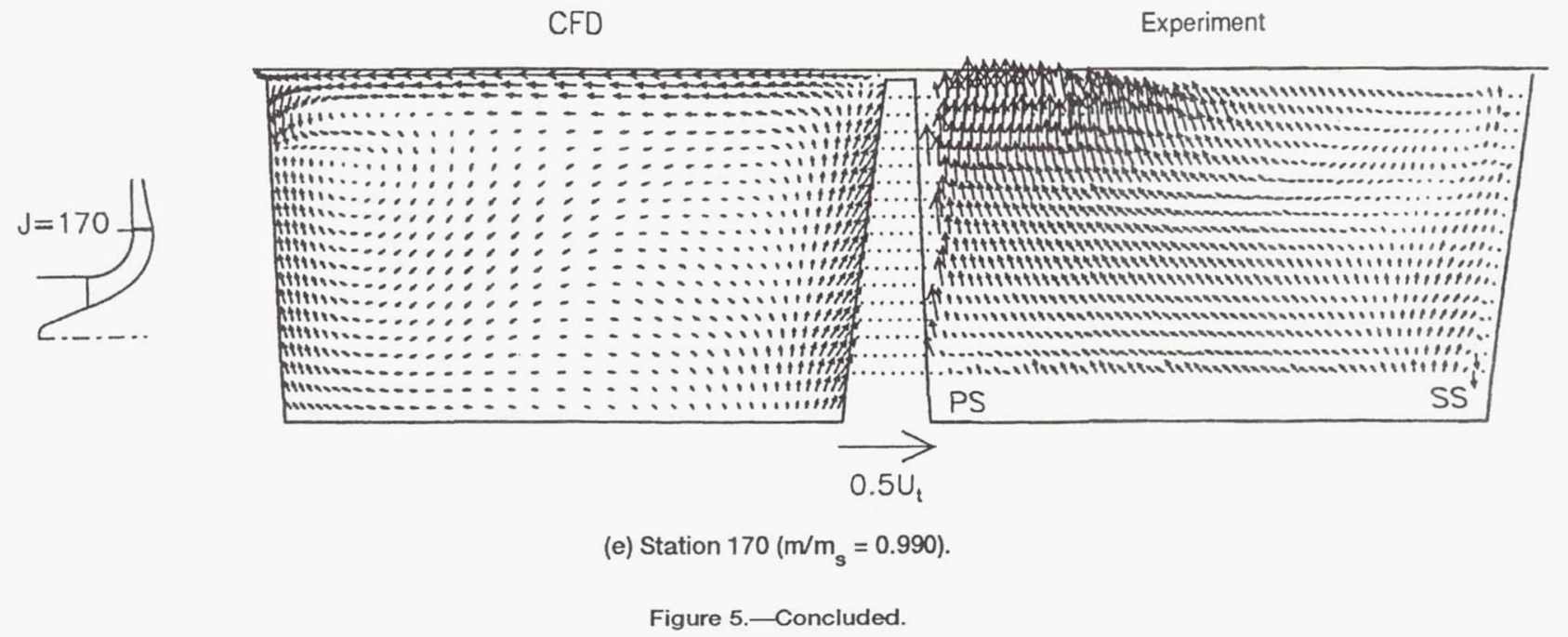

tip near the blade surfaces. However, the CFD results indicate that the fluid migrating outward along the blade pressure surface is now being met and turned inward by fluid moving downward near the blade pressure surface/shroud corner, just as was shown in the experimental results at station 118. In addition, the CFD tracers show further migration toward the blade pressure surface and they appear to be rolling up into a vortical flow. The experimental results now show a stronger vortical flow near the shroud than that indicated at station 118 .

The insets in figure 5(c) show additional details of the measured and predicted flows in the tip region which, for the sake of clarity, are not provided in the full-size passage view. The vector scale is different for each inset and is chosen so as to minimize overlap among vectors. Details of the predicted results in the blade pressure surface/shroud corner are shown in the left inset of figure 5(c), which is a magnified view of the full-size passage results. The results show that the inward flow in the pressure surface/shroud corner of the passage is caused by the rollup of endwall fluid near the tip of the blade. The middle inset of figure 5(c) provides further details of the measured flow field from 95 to 100 percent of span at the full pitchwise resolution of the laser anemometer measurements. The results verify the rollup of fluid predicted by the CFD solution in the pressure surface/shroud corner and show the movement of fluid near the suction surface into the tip clearance gap. Finally, the right inset provides details measured within the blade suction surface boundary layer at 85,90 , and 95 percent of span at the full pitchwise resolution of the laser anemometer measurements. The data clearly show the migration of the suction surface boundary layer fluid toward the shroud.

A comparison of figures 3(c) and 5(c) indicates that there is once again a strong correlation between the location of the throughflow velocity deficit and the location of strong secondary flow. As shown in figure 3(c), the magnitude of the measured normalized throughflow velocity in the pres- sure surface/shroud corner of the passage is $V_{q m} / U_{t}=0.10$ to 0.15 . In this region the magnitude of the secondary flow is on a par with the throughflow velocity.

At station 165 (shown in fig. 5(d)), the CFD solution indicates a continued migration of flow outward along the blade surfaces and a well-behaved rollup of flow in the pressure surface/shroud corner of the passage. The experimental results do not indicate outflow along the suction surface of the blade as predicted by the CFD result. We also note that a weak but discernible outward flow appears in the measurements towards the pressure-side half of the passage below about 70 percent of span. However, the most dominant measured flow features are the strong spanwise flows in the pressure surface/shroud corner with an apparent strong reversal in spanwise flow direction (to be addressed subsequently) at about 87 percent of span. The measurements in this region were repeated on three separate occasions and each measurement session yielded identical results.

At station 170 (shown in fig. 5(e)), we see a dramatic change in the measured secondary flow relative to that just shown at station 165 . The outward flow near the blade pressure surface, which was located below about 70 percent of span and was quite weak at station 165 , now dominates the secondary flow field in the pressure surface/shroud corner.

To investigate the cause of the secondary flow results shown in figure 5(d), we inspected the statistics of the individual laser anemometer measurements acquired at each of the 200 measurement points across the blade pitch. At each point a probability density distribution (p.d.d.) was constructed for all the measurements which occurred at that point. Throughout most of the flow field, these p.d.d.'s are Gaussian in character, which is to be expected for a turbulent flow. However, for points in the pressure surface/shroud corner at station 165, the p.d.d.'s are skewed, with long "tails" that result from the occurrence of velocities that are well below the mean velocity. The averaging method used to process all the laser anemometer data essentially calculates the 


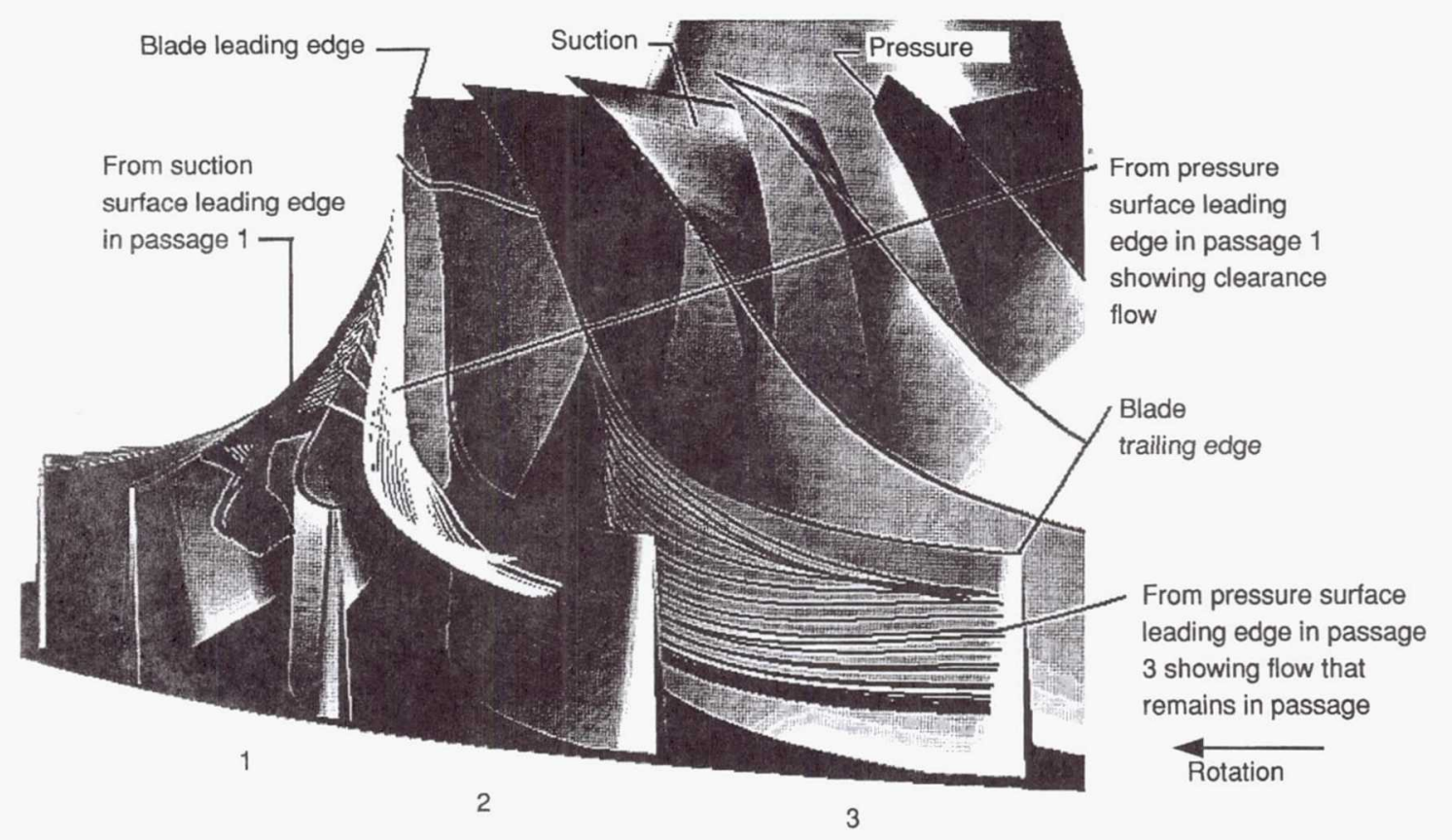

Figure 6.-Perspective view of the paths of CFD tracers released on blade surfaces near impeller leading edge.

mean of each p.d.d. For Gaussian p.d.d.'s the mode, defined as the velocity which occurs most often, is close to or identical to the mean. However, for the p.d.d.'s that are skewed toward lower velocities, the mean occurs at a lower velocity than the mode.

The secondary velocity vectors shown in the inset in figure 5(d) are calculated using the mode of each p.d.d. rather than the mean. The mode results yield a secondary velocity field which has no vortical structure and is more similar to that measured at station 170. Since the mode is by definition the velocity which occurs most often, the mode results are felt to be more representative of the secondary velocity field in the pressure surface/shroud corner at station 165.

The skewed p.d.d.'s in the pressure surface/shroud corner suggest that the flow is quasi-steady in this region. This might be caused by a meandering of the throughflow wake at station 165 . The secondary velocity vectors at stations 135 and 170 were also calculated based on the mode of the p.d.d.'s. The results, which are not included here, indicate that there is no appreciable difference between the vectors calculated using the mode and those calculated using the mean. These results indicate that the flow is steady at stations 135 and 170.

The results at station 135 indicate the development of a vortex which does not appear at stations 165 and 170. The ability to "see" a vortex is highly dependent on the viewing direction used when creating the secondary velocity vectors. The fact that a vortex appears at station 135 indicates that the vortex core is well aligned with the streamwise grid lines at that station. The results shown in figure 3 indicate that the throughflow velocity wake drifts from a location near the pressure surface toward midpitch as we move from station
135 to stations 165 and 170 . If the vortex is associated with the wake, then the vortex core might also drift toward midpitch in the rear of the rotor. The vortex might therefore still be present at stations 165 and 170 but is no longer evident in the secondary velocity vector plots because the core is no longer aligned with the streamwise grid lines.

The secondary velocity results and CFD particle tracers presented in figure 5 illustrate that low-momentum fluid near the blade surface migrates toward the blade tip throughout most of the impeller passage, becomes entrained in the tip clearance flow, and then migrates toward the blade pressure surface/shroud corner. A perspective view of this process is presented in figure 6 , which shows the predicted trajectories of particles that are released from the hub and tip along the blade leading edge on either side of the leading edge stagnation line. The view in figure 6 is looking upstream into the impeller blade passage from a viewpoint near the impeller exit.

The black traces in passage 1 are the trajectories of particles released on the suction surface in blade passage 1 . These particles migrate up the blade surface toward the tip, are entrained in the tip clearance jet and are transported across the blade passage near the shroud, and then begin to roll up in the pressure surface/shroud corner of passage 1 . This rollup can be clearly seen in figure 6 and also in both the CFD solution and the laser measurements at station 135 (fig. 5(c)). The white traces in figure 6 are the trajectories of particles released in blade passage 1 on the pressure surface outboard of 60 percent of span. These particles migrate toward the tip, cross over the blade, move across the passage near the shroud, and roll up in the pressure surface/shroud corner in passage 2 . The migration of pressure surface fluid 
across the tip can also be seen in the CFD solution at stations 85 and 118 (figs. 5(a) and (b)). The black traces in passage 3 in figure 6 are the trajectories of particles released in blade passage 3 on the pressure surface inboard of 60 percent of span. These particles begin to migrate toward the tip but are met and turned inward by the fluid that rolls up in the pressure surface/shroud corner. This behavior can also be seen in the CFD results shown in figures 5(c) and (d) at stations 135 and 165. A comparison of the particle trajectories in figure 6 with the throughflow velocity results of figure 3 clearly demonstrates that the throughflow velocity wake is formed by the migration of low-momentum fluid near the blade surfaces toward the shroud where it is entrained by the tip clearance flow and transported toward the pressure surface.

We have studied the trajectories of additional CFD tracers released all along the blade chord on the blade surfaces near the tip. These trajectories, which are not shown here, indicate that all the fluid outboard of the tracers shown in figure 5 originated on the blade surfaces. The tracer locations shown in this figure therefore define the lower bound of the tip clearance flow.

To supplement the laser anemometer secondary flow measurements near the blade surfaces, the ammonia-ozalid surface flow visualization technique described by Joslyn and Dring (1987) was used to measure the flow direction along the impeller blade suction and pressure surfaces. The uncertainty in the surface flow angles measured with this technique is on the order of $2^{\circ}$.

The results of the ammonia-ozalid technique are shown in figure 7 . The suction surface flow traces show a significant spanwise flow toward the tip in the area between stations 85 and 135. The ammonia traces at station $85\left(\mathrm{~m} / \mathrm{m}_{\mathrm{s}}=0.149\right)$ indicate that fluid is already entering the tip clearance gap at this station. An appreciable accumulation of seed particles was observed on the shroud windows in the region $\mathrm{m} / \mathrm{m}_{s}=0.20$ to 0.30 , which provides further evidence that the clearance jet develops to an appreciable strength in this region.

The ammonia traces near station 118 indicate a strong outward flow along the entire suction surface. The ammonia traces along the pressure surface indicate an outward flow below midspan which diminishes in strength and then becomes an inward flow as we move toward the tip. The measured velocity vector results at station 118 (shown in fig. 5(b)), are in qualitative agreement with these ammoniaozalid results. The ammonia traces on the pressure surface near stations 135 and 165 show several reversals from spanwise inward to spanwise outward flow in the outer half of the blade span. These changes in surface flow direction are also in qualitative agreement with the measured velocity vector results shown in figures $5(\mathrm{c})$ and (d), which indicate the presence of a strong secondary flow in the pressure surface/ shroud corner of the blade passage. The velocity vector results at station 170 (fig. 5(e)) do not seem to agree with the ammonia trace results on the pressure surface near the shroud. The ammonia results show that the flow near the shroud is moving inward whereas the velocity vector results seem to indicate that the flow is moving outward at all spanwise locations near the pressure surface. This apparent discrepancy arises from the fact that we were not able to measure the velocity near the pressure surface at station 170 because of an optical blockage problem.

The predicted flow directions near the blade pressure surface in the rear of the impeller are shown in figure 8 , which is a meridional view of the CFD results on the first grid plane from the blade surface. A demarcation line that separates flow moving toward the hub from that moving toward the tip can be seen at about 80 percent of span near the impeller exit. This demarcation line can also be seen in figure 6 for the CFD tracer results near the pressure surface in passage 3 . The

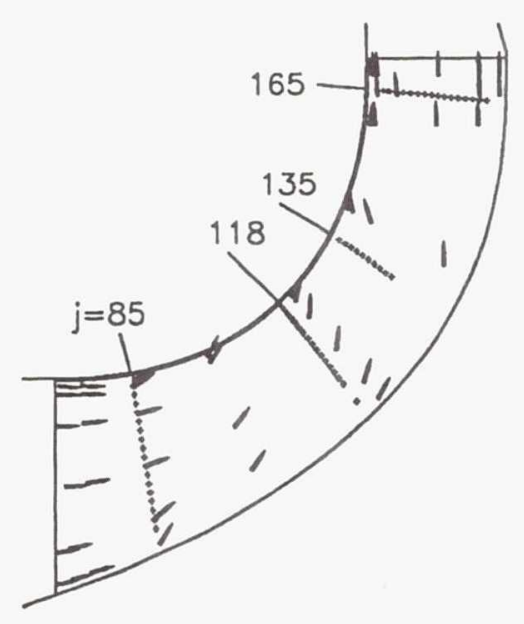

(a) Suction surface.

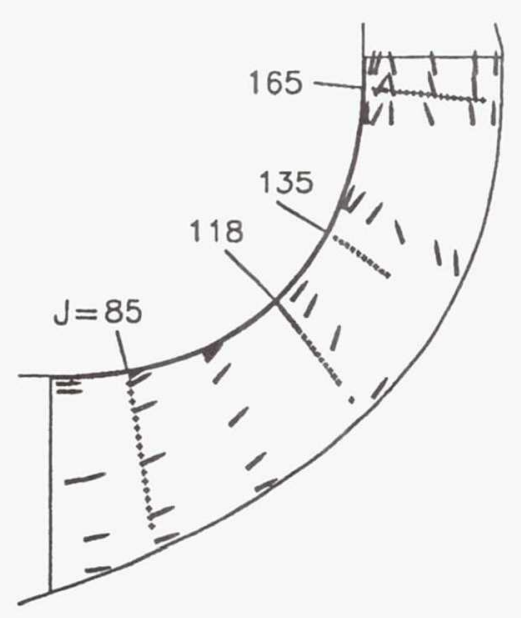

(b) Pressure surface.

Figure 7.-Meridional view of suction and pressure surface flow directions measured using ammonia-ozalid flow visualization. 


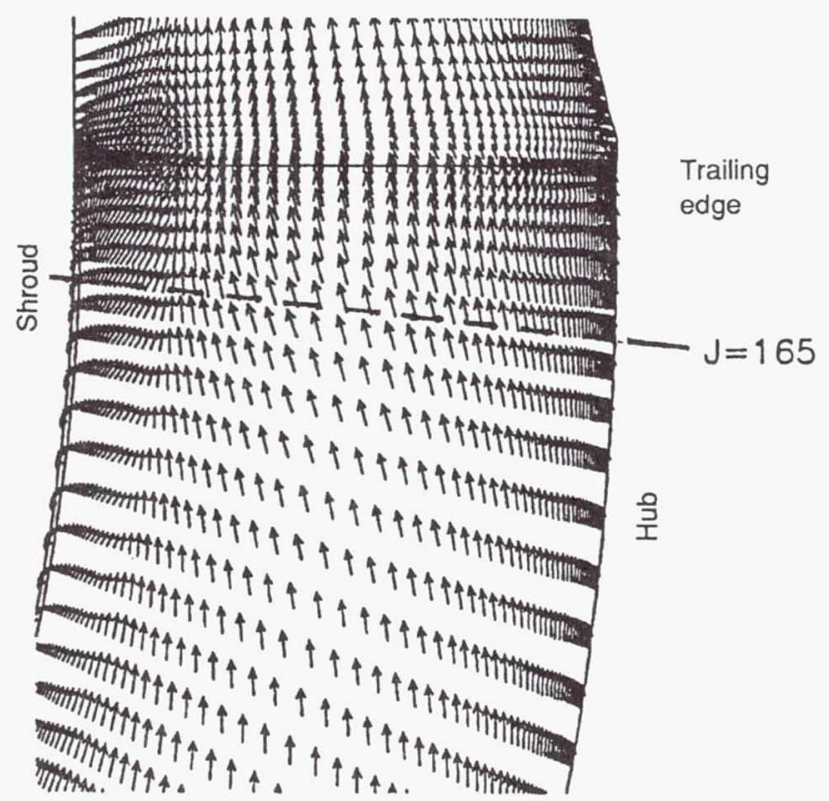

Figure 8.-Meridional view of predicted velocity vectors near the pressure surface.

CFD results are in excellent qualitative agreement with the pressure surface ammonia-ozalid results shown in figure 7 (b) at the impeller exit near the tip; these results also indicate the presence of a distinct change in spanwise flow at about 80 percent of span.

The secondary flow field results presented thus far have qualitatively illustrated the features of the secondary flow field and the comparison between CFD and experimental results. A more quantitative comparison between CFD and experimental results will now be provided by presenting blade-to-blade distributions of velocity and pitch angle at selected measurement locations at stations 118 and 165.

The measured and predicted distributions of the normalized quasi-meridional velocity, normalized spanwise secondary velocity, and pitch angle at 65 percent of span at station 118 are shown in figure 9 . The comparison between predicted and measured values of $V_{q m} / U_{t}$, which approximates the throughflow velocity, is excellent. The extent of the suction and pressure surface boundary layers is clearly shown. The laser anemometer measurements of the normalized spanwise secondary velocity $V_{s} / U_{\mathrm{t}}$ (see fig. $9(\mathrm{~b})$ ) indicate appreciable outflow near both the pressure and suction surfaces and a weak inflow across the remainder of the passage. Note that the outflow regions shown in figure 9 (b) closely correspond to the blade boundary layers shown in figure 9(a). The ability of the laser measurements to accurately resolve the spanwise velocity component is illustrated in figure 9(c) where we see that the pitch angles $\alpha$ measured on the blade surfaces with the ammonia-ozalid flow visualization technique are in close agreement with the near-surface pitch angles measured with the laser anemometer.

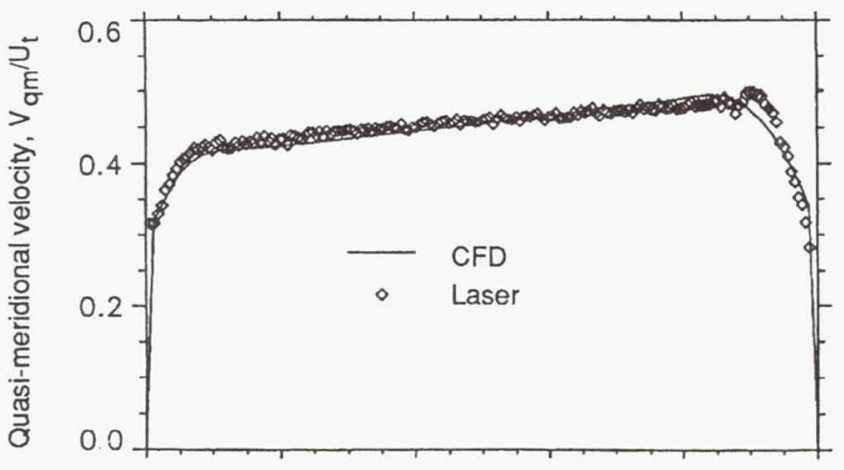

(a) Quasi-meridional velocity.

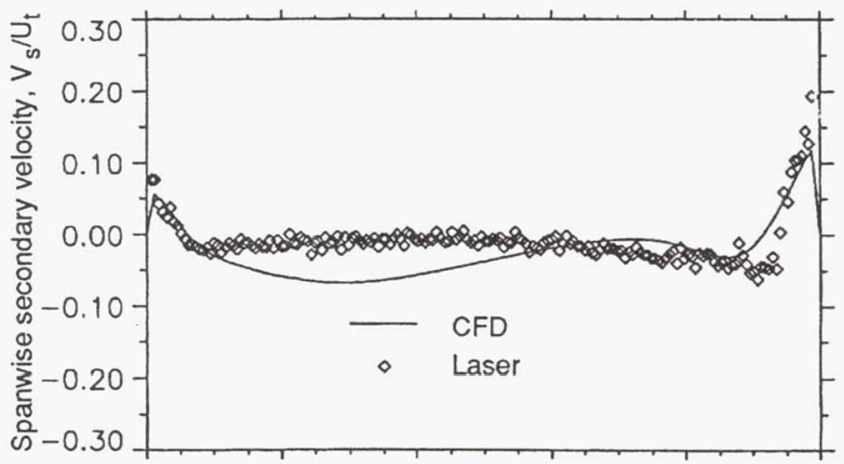

(b) Spanwise secondary velocity.

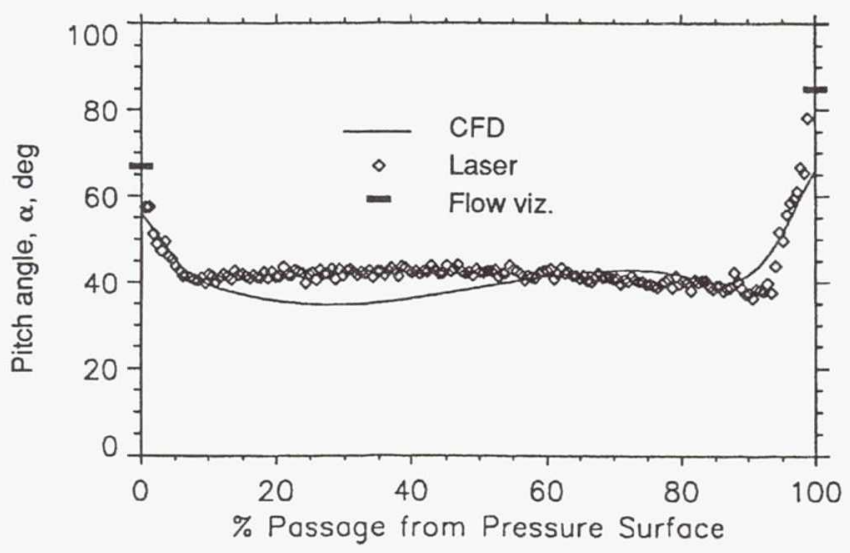

(c) Pitch angle.

Figure 9.-Blade-to-blade distribution of quasi-meridional and spanwise secondary velocities and pitch angle at 65 percent of span for station $118\left(\mathrm{~m} / \mathrm{m}_{\mathrm{s}}=0.475\right)$.

Measured and predicted pitch angle distributions as well as the ammonia-ozalid surface flow angle results at 20,80, and 95 percent of span for station 165 are shown in figure 10. At 80 and 95 percent of span, the pitch angle distributions derived from both the mean and mode of the laser measurements are shown. As discussed previously, the mode results are considered more representative of the flow in this region 
than are the mean results. The grid normal at station 165 has a pitch angle of $85^{\circ}$. Therefore, a flow pitch angle which is greater than $85^{\circ}$ indicates a spanwise flow toward the shroud whereas a flow pitch angle which is less than $85^{\circ}$ indicates a spanwise flow toward the hub.

There is no appreciable spanwise flow across the entire passage at the 20 -percent span location. The laser measurements do not indicate a large change in flow angle near the

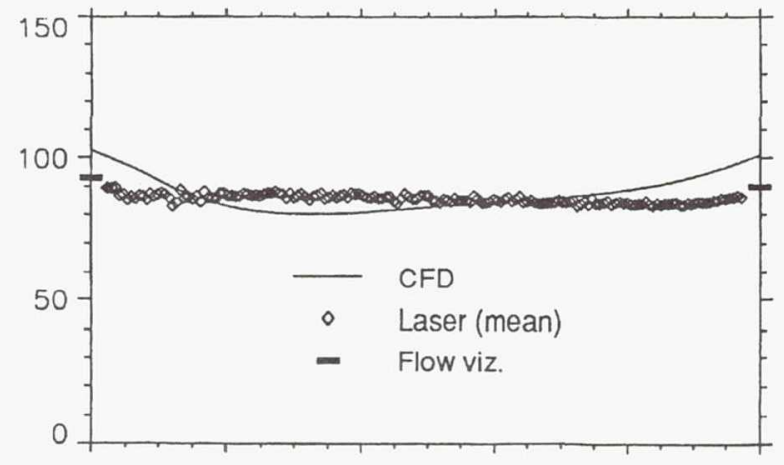

(a) Twenty percent.

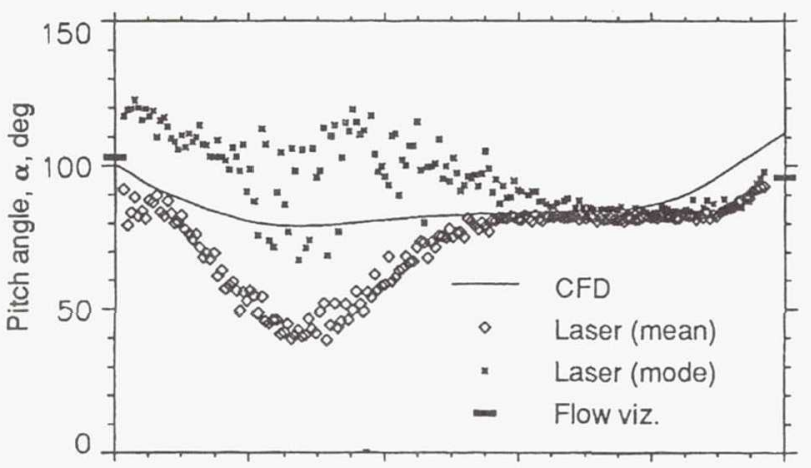

(b) Eighty percent.

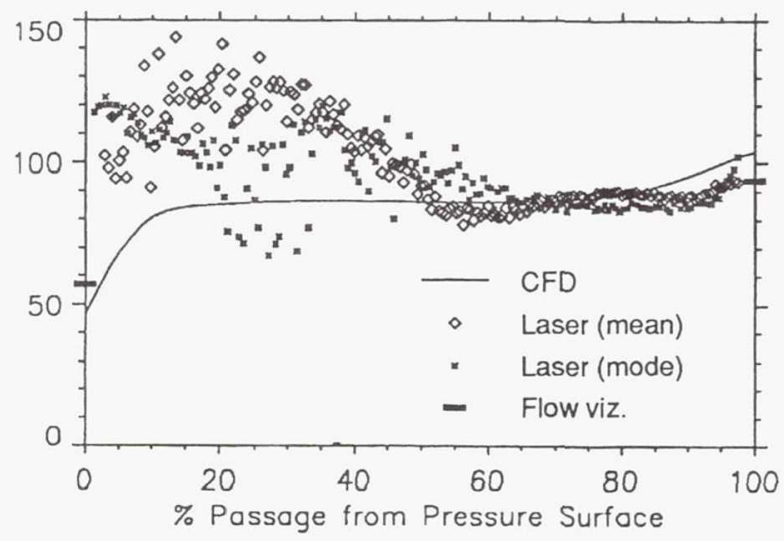

(c) Ninety-five percent.

Figure 10.-Blade-to-blade distribution of pitch angle at 20, 80 , and 95 percent of span for station $165\left(\mathrm{~m} / \mathrm{m}_{\mathbf{3}}=0.941\right)$. pressure surface between 80 and 95 percent of span. However, the CFD and ammonia-ozalid results indicate a change in pitch angle on the pressure surface on the order of $50^{\circ}$ between these two spanwise locations. Note that all three techniques also indicate a relatively weak spanwise flow along the suction surface at all immersions.

\section{Impeller Wake Mixing}

Mixing phenomena downstream of centrifugal impellers are quite complex. At the impeller exit a viscous blade wake is present at all spans, and a throughflow velocity wake is
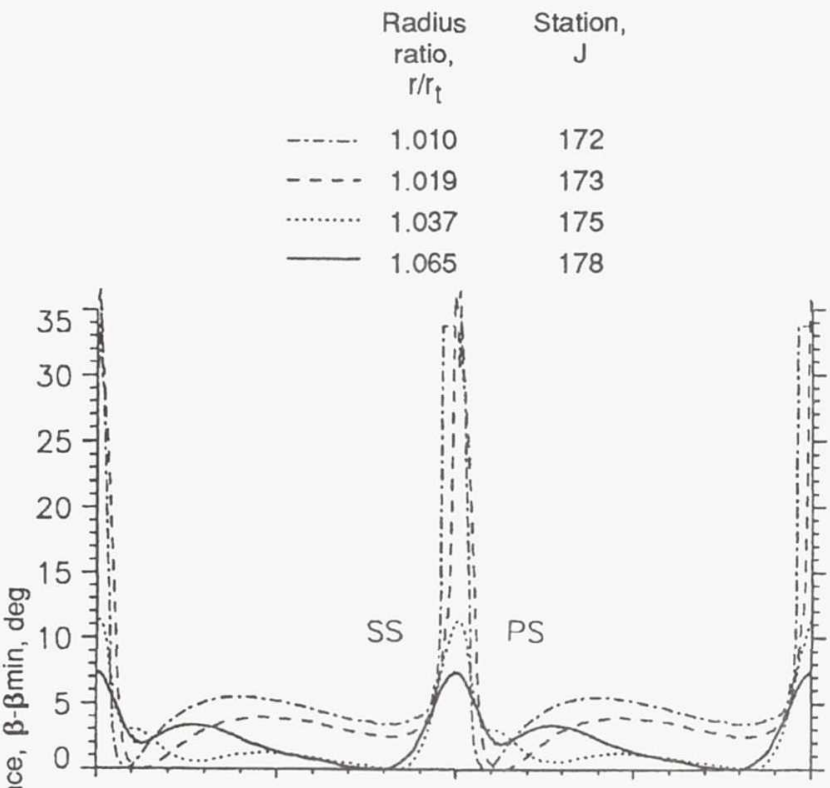

(a) Fifty percent span.

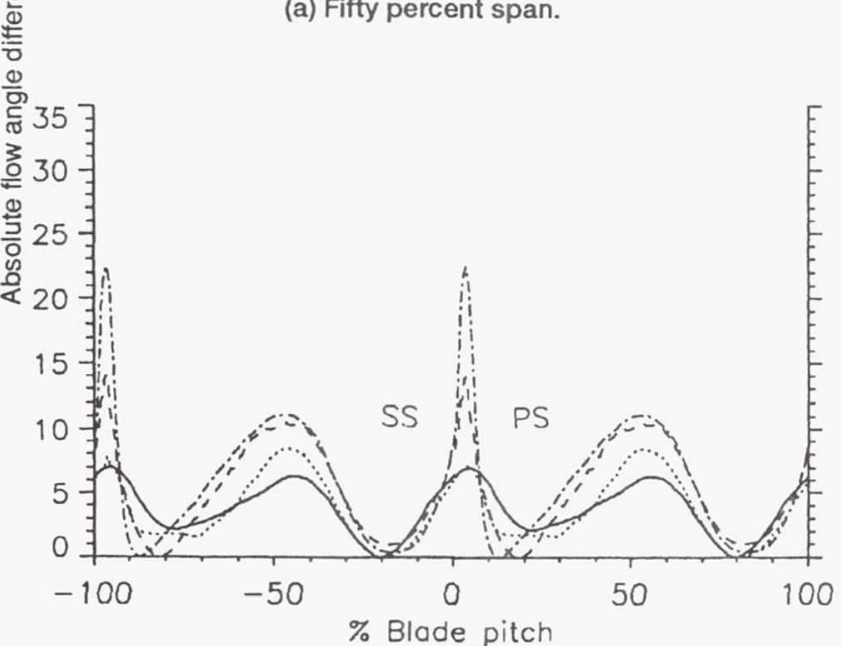

(b) Ninety percent span.

Figure 11.-Pitchwise distribution of absolute flow angle difference, $\boldsymbol{\beta}-\boldsymbol{\beta}_{\min }$ in vaneless diffuser at four measurement stations downstream of the impeller trailing edge. 


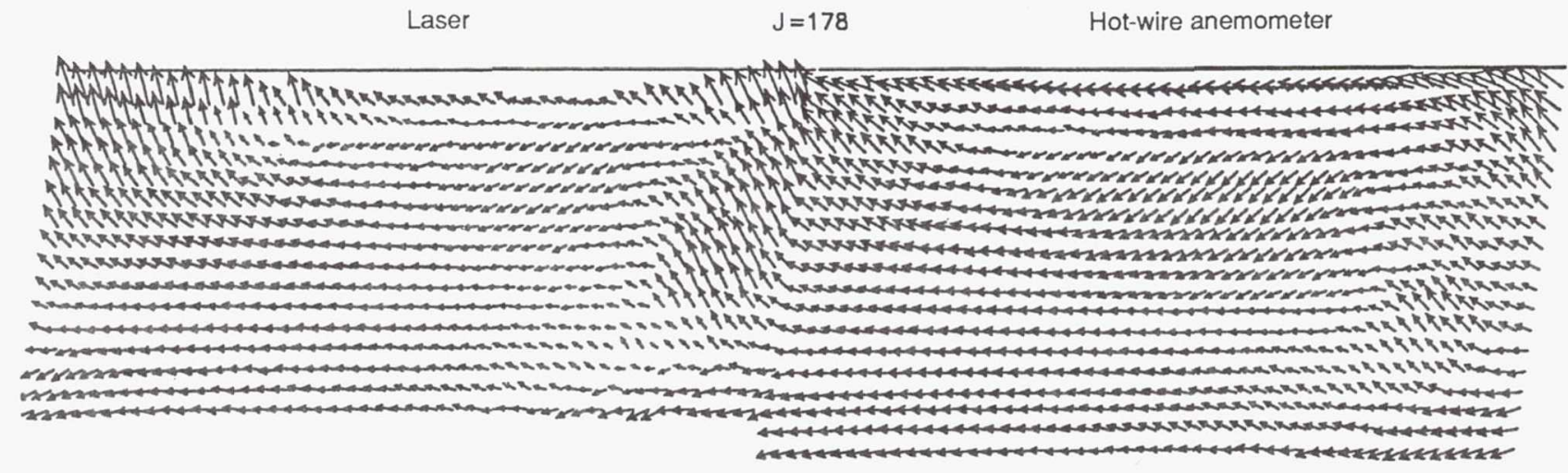

Figure 12.-Comparison of laser and hot-wire anemometer measurements of secondary velocity vectors at station 178 (radius ratio, $r / r_{\mathrm{t}}=1.065$ ).

present near midpitch in the outer 30 percent of the blade span. To study wake mixing phenomena, laser anemometer measurements were acquired at four measurement stations in the vaneless diffuser at radius ratios of 1.010, 1.019, 1.037, and 1.065 . The absolute flow angle distributions acquired at these stations are used to illustrate the mixing in the LSCC vaneless space. The results provide an indication of the incidence angle variation in the diffuser vanes if a vaned diffuser were present.

The blade-to-blade variation in the absolute flow angle at 50 percent of span is presented in figure 11(a) for the four measurement stations in the vaneless diffuser. The results are shown across two complete blade pitches, with the blade centered at 0 percent blade pitch. For each radius ratio, the flow angle variation is presented as the difference between the local flow angle $\beta$ and the minimum flow angle at that radius ratio $\beta_{\min }$. At radius ratios of 1.010 and 1.019 , the flow angle variation across the viscous blade wake is more than $30^{\circ}$. This angle variation decreases rapidly to levels below $10^{\circ}$ at radius ratios of 1.037 and 1.065 . The flow angle variation across the remainder of the blade passage is less than $6^{\circ}$ at all radius ratios.

The pitchwise variation in the flow angle at 90 percent of span is shown in figure 11(b). At this spanwise location, the flow exiting the impeller contains a throughflow velocity wake as well as a viscous blade wake. The blade is thinner at 90 percent of span than at 50 percent of span, which contributes to a weaker viscous blade wake than at 50 percent of span. At a radius ratio of 1.010, the absolute angle variation across the viscous wake is on the order of $20^{\circ}$ whereas the angle variation across the throughflow velocity wake is on the order of $10^{\circ}$. As we move downstream, the flow angle variation across the viscous wake decreases rapidly. However, the angle variation across the throughflow wake decreases much more slowly. As a result, the variation in the absolute angle across the viscous and throughflow velocity wakes is almost equal at radius ratios of 1.037 and 1.065 .
Hot-wire anemometer surveys of the flow field were acquired at a radius ratio of 1.065 using a slanted hot-wire measurement technique described by Hanson et al. (1992). The primary purpose of this exercise was to provide an independent check on the accuracy of the laser anemometer measurement technique. The hot-wire and laser anemometer results are shown in figure 12 . Both measurements indicate flow migrating towards the shroud in the viscous wake region (towards the right side of each plot) and in the throughflow wake region (towards the left side of each plot over the upper 50 percent immersion).

\section{Fidelity of Low-Speed Compressor Flow Field Simulation}

The LSCC was designed to generate a flow field which is aerodynamically similar to that found in high-speed subsonic centrifugal compressors, as described by Wood, Adam, and Buggele (1983) and by Hathaway, Wood, and Wasserbauer (1992). If the flow field that has been investigated in the LSCC can be shown to indeed be similar to that found in actual subsonic centrifugal compressors, then the results presented herein can be used to assess the accuracy of NavierStokes analyses and the lessons learned can be used with confidence when analyzing actual high-speed impellers. To determine how well the LSCC flow field simulated that found in a high-speed subsonic compressor, the throughflow velocity in the LSCC was compared with the throughflow velocity measured in two 4 -to- 1 pressure ratio, $30^{\circ}$ backswept impellers as reported by Krain and Hoffman (1989 and 1990). These impellers were denoted rotors I and II. Rotor II is similar to rotor I but features a reduction in the exit blade height of approximately 30 percent. The tip speed of rotor I is $470 \mathrm{~m} / \mathrm{sec}$ and the tip clearance is $0.2 \mathrm{~mm}$ at the exit and $0.5 \mathrm{~mm}$ at the inlet. The exit blade span of the impeller is $14.7 \mathrm{~mm}$, which yields a tip clearance based on exit blade 


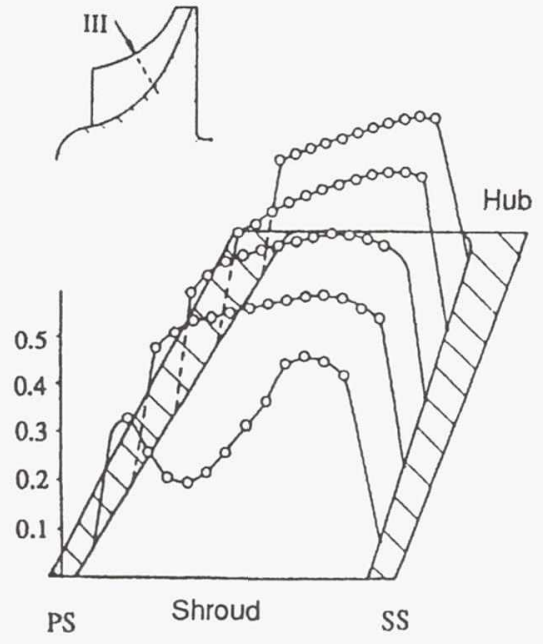

(a) Rotor I, measurement plane III $\left(\mathrm{m} / \mathrm{m}_{3}=0.4\right)$.

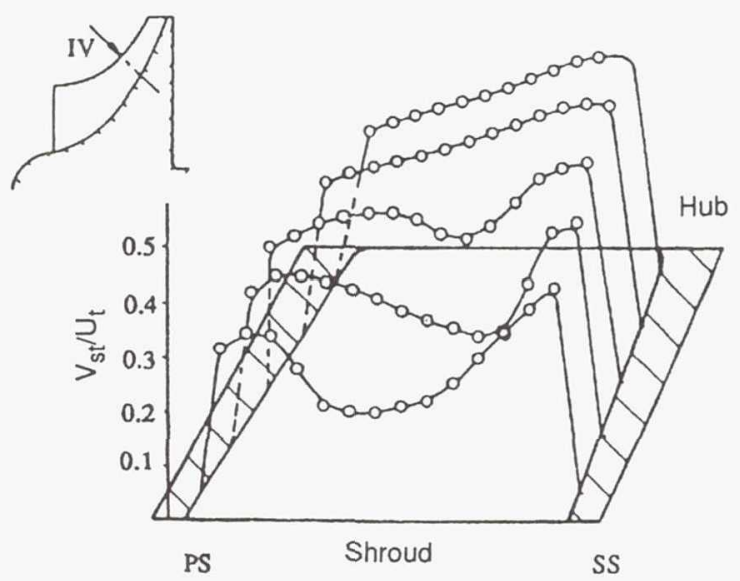

(b) Rotor I, measurement plane IV $\left(\mathrm{m} / \mathrm{m}_{\mathrm{s}}=0.6\right)$.

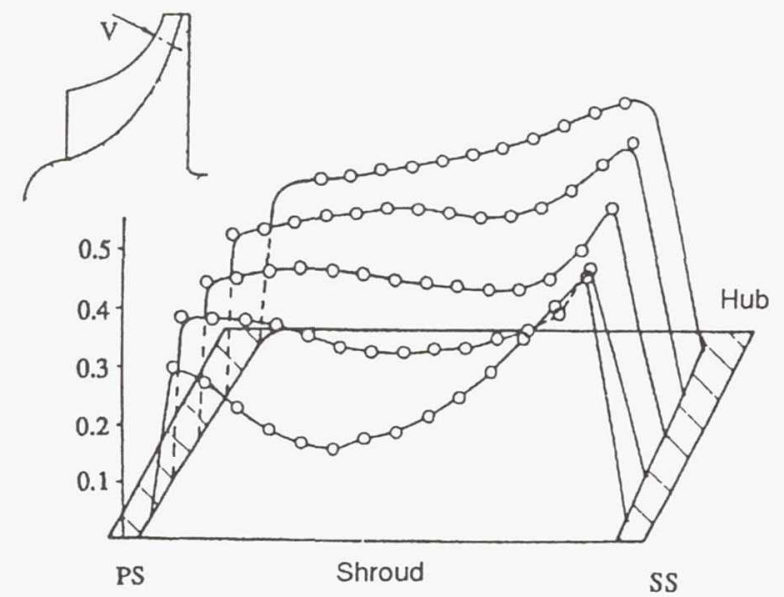

(c) Rotor I, measurement plane $\mathrm{V}\left(\mathrm{m} / \mathrm{m}_{\mathrm{s}}=0.8\right)$.

Figure 13.-Throughflow velocity measurements in high-speed (4:1 pressure ratio) centrifugal compressor (Krain and Hoffman, 1989, 1990).
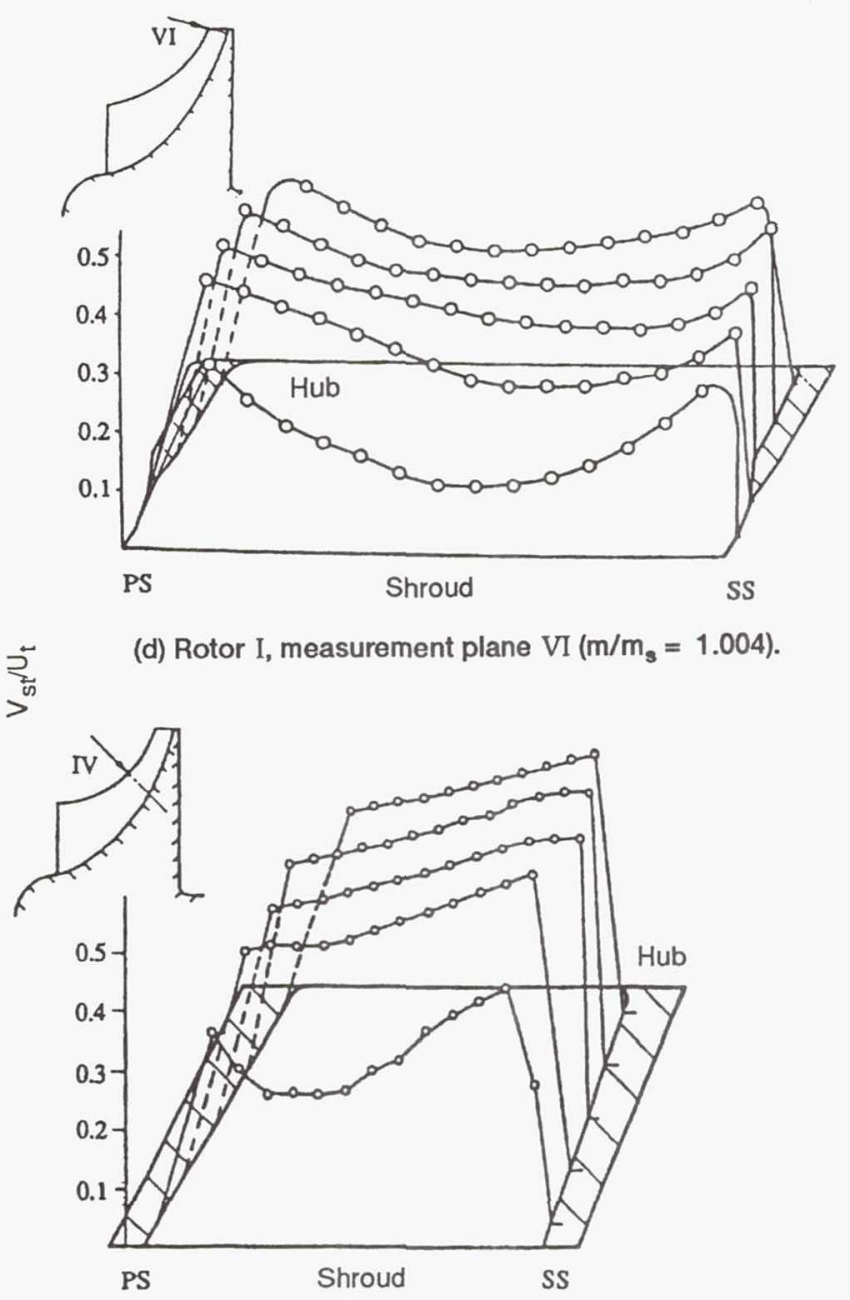

(e) Rotor II, measurement plane IV $\left(\mathrm{m} / \mathrm{m}_{\mathrm{s}}=0.6\right)$.

Figure 13.-Concluded.

span of 1.4 percent $(0.2 \mathrm{~mm})$ at the exit and 3.4 percent $(0.5 \mathrm{~mm})$ at the inlet. The tip clearance of the LSCC based on the exit blade span is 1.8 percent $(2.54 \mathrm{~mm})$ and is constant from inlet to exit.

The LSCC and rotor I and II results are shown in figures 3 and 13, respectively. The rotor I and II results are for 10, 30 , 50,70 , and 90 percent of span at each measurement plane (herein referred to as station) whereas the LSCC results are for every 5 percent of span starting at 95 percent of span. The velocity component measured by Krain and Hoffman, denoted herein as $V_{s t}$, is tangent to the shroud in the meridional direction. The velocity component measured in the LSCC, $V_{q m}$, is tangent to the local streamwise grid lines, which are tangent to the shroud at 100 percent of blade span and tangent to the hub at 0 percent of blade span. Although $V_{s t}$ and $V_{q m}$ are not identical velocity components, they are very similar. 
The throughflow velocity wake first appears in rotor I results near the pressure surface at station III and occupies at least 10 percent of span at this station. This result compares favorably with that measured in the LSCC at station 118 (fig. 3(b)). The LSCC results at station 135, however, do not agree with rotor I results at station IV. The wake in the LSCC is close to the blade pressure surface at this station whereas the wake in rotor I remains near midpitch. The wake in rotor I penetrates to 50 percent of span whereas that in the LSCC only penetrates to 75 percent of span, which can be attributed to the tip clearance in the inlet region of rotor I being more than double that of the LSCC. In moving from station $\mathrm{V}$ to VI in rotor I, the velocity near the pressure surface rises while the velocity near the suction surface falls, indicating a reduction in blade loading. This behavior is similar to that found in the LSCC as we proceed from stations 165 to 170 .

The movement of the rotor I wake toward midpitch at station IV might result from the low-momentum fluid in the wake being subjected to a larger cross-passage pressure gradient than that in the LSCC. This may indeed be what is occurring if the results from rotor II (Krain and Hoffman, 1990) are considered. Because the throughflow velocity in rotor II is somewhat higher than that in rotor I, whereas the cross-passage pressure gradient and the overall pressure ratio are similar for both rotors, it is reasonable to expect that the low-momentum wake fluid in rotor II would remain closer to the pressure side of the passage than the wake fluid in rotor I. The plane IV results measured in rotor II (fig. 13(e)) confirm that the wake is located closer to the pressure surface than it is in rotor I. In addition, the wake in rotor II does not penetrate below 70 percent of span, which agrees favorably with the wake penetration to 75 percent of span in the LSCC at station 135 .

An analysis of rotors I and II and the LSCC with a one-dimensional, centrifugal compressor performance prediction code shows that the relative velocity ratio (exit velocity/inlet tip velocity) of the LSCC falls between that of rotors I and II.

In summary, the throughflow development in the LSCC impeller is similar to that in Krain's impellers. Differences between the LSCC throughflow characteristics and those in rotors I and II are on the same order as the differences between the two high-speed rotors. Since the throughflow development is tightly coupled to the secondary flow development, the secondary flow field characteristics in the LSCC should indeed be similar to those which exist in high-speed subsonic centrifugal impellers.

\section{Conclusions}

The results presented herein represent an extensive computational and experimental effort to document the velocity field within the NASA Lewis low-speed centrifugal compressor (LSCC). The large impeller passage size provides considerable optical access to the flow field and enables measurements of the three-dimensional velocity field throughout the impeller blade passage. In several cases the measurements provide details within the blade boundary layers. Independent validation of the laser measurements via five-hole probe and hot-wire anemometer surveys as well as ammonia-ozalid surface flow visualization results provides a high degree of confidence in the laser anemometer results.

The laser anemometer measurements and the CFD solution complement one another in illustrating the flow physics within the impeller. Laser anemometer measurements confirm that the low-momentum fluid near the blade surfaces migrates outward toward the tip of the blade as predicted by the CFD solution. Particle traces done on the CFD solution indicate that the fluid which moves up the blade pressure and suction surfaces is entrained in the tip clearance jet. The particle traces also show that this fluid is then transported toward the pressure side/shroud corner of the passage where it contributes to the formation of the characteristic throughflow momentum wake that is found in unshrouded centrifugal compressor impellers.

The throughflow momentum wake region that is generated as a result of the tip clearance flow mixes out more slowly than does the viscous blade wake as the flow moves through the vaneless diffuser. The flow field in the vaneless space near the shroud surface is therefore composed of two lowmomentum regions - one from the viscous blade wake and one from the throughflow momentum wake.

The development of the throughflow velocity field within the LSCC is shown to be qualitatively similar to that measured in a high-speed, 4-to-1 pressure ratio centrifugal compressor. The experimental results presented provide a representative view of the flow physics within unshrouded centrifugal compressor impellers and should be useful in assessing the ability of viscous flow codes to accurately predict the flow physics within centrifugal compressors.

Lewis Research Center

National Aeronautics and Space Administration

Cleveland, Ohio, July 13, 1993

\section{Acknowledgments}

The authors would like to acknowledge those without whose support this research would not have been possible: Bill Darby, Bob Davis, John DeArmon, Marty Pietrasz, Rick Senyitko, Charles Wasserbauer, and Hal Weaver for their support of the LSCC experiment; Glenn Christman, Tim Hepner, and Mark Wernet for their support and advice relative to laser anemometer hardware and software; and Bruce Reichert, Kurt Hansen, and Andy Hiedinger for acquiring the hot-wire anemometer results. 


\section{Appendix-Definition of Velocity Components}

To visualize secondary flow, the total relative velocity vector must be viewed along the streamwise direction. However, in a geometrically complex channel such as a centrifugal impeller blade passage, the streamwise direction can be defined in several different ways, and each definition will yield a slightly different result for the secondary flow. This problem has been pointed out by many previous researchers. The purpose of the following discussion is to document the procedure used to generate the secondary flow field results presented in this report. The same procedure is applied to both the computational and experimental results. The secondary flows presented herein are defined as the departure of the local relative velocity vector from the local streamwise grid direction. The secondary velocity vector is given by $\vec{V}_{T}-\vec{V}_{\mathrm{g}}$, where $\vec{V}_{T}$ is the relative velocity vector and $\vec{V}_{\mathrm{g}}$ is the projection of $\vec{V}_{T}$ in the local streamwise grid direction $\bar{g}(r, \theta, z)$. The spanwise and pitchwise components of the secondary velocity vector, $V_{s}$ and $V_{p}$, are the projections of the secondary velocity vector in the local spanwise and pitchwise grid directions, as given by the two dot products

$$
\begin{aligned}
V_{s} & =\left(\vec{V}_{T}-\vec{V}_{g}\right) \cdot \hat{g}_{s} \\
V_{p} & =\left(\vec{V}_{T}-\vec{V}_{g}\right) \cdot \hat{g}_{p}
\end{aligned}
$$

where $\hat{g}_{s}$ and $\hat{g}_{p}$ are unit vectors in the local spanwise and pitchwise grid directions. When secondary flow results are presented in the form of vector plots in a quasi-orthogonal plane, $V_{s}$ and $V_{p}$ are used to determine the magnitude and direction of the plotted secondary velocity vectors.

The procedure just described is applied at each measurement grid node when processing experimental data and at each CFD grid node when processing CFD results. Thus, a flow field with no secondary flow components will appear as a point at each grid node, indicating that the flow is following the streamwise grid direction. Since the local streamwise grid direction is parallel to the blade, hub, and shroud surfaces, the definition of secondary flow presented herein also insures that the secondary velocity be zero at all solid surfaces.

The quasi-meridional velocity component $V_{q m}$ is the vector projection of the meridional velocity vector $\vec{V}_{m}$ in the local streamwise grid direction $V_{q m}=\vec{V}_{m} \cdot \hat{g}_{m}$. Since a quasi-orthogonal plane is nearly normal to the streamwise grid direction in the meridional plane at any station in the impeller, $V_{q m}$ is a close approximation to the throughflow velocity which crosses a quasi-orthogonal plane; $V_{q m}$ is also a close approximation to the streamwise velocity component measured in laser anemometer investigations published by previous authors (Krain, 1988; Ahmed and Elder, 1990; Fagan and Fleeter, 1991). In these investigations, the streamwise velocity component was defined as the velocity component in the direction tangent to the shroud meridional direction at each measurement station.

\section{References}

Ahmed, N. A.; and Elder, R. L.: Flow Investigation in a Small High Speed Impeller Passage Using Laser Anemometry. ASME Paper 90-GT-233, 1990.

Baldwin, B.; and Lomax, H.: Thin Layer Approximation and Algebraic Model for Separated Turbulent Flows. AIAA Paper 78-257, 1978.

Bansod, P.; and Rhie, C. M.: Computation of Flow Through a Centrifugal Impeller with Tip Leakage. AIAA Paper 90-2021,1990.

Dawes, W. N.: Development of a 3-D Navier Stokes Solver for Application to all Types of Turbomachinery. ASME Paper 88-GT-70, 1988.

Eckardt, D.: Detailed Flow Investigations Within a High-Speed Centrifugal Compressor Impeller. J. Fluids Eng., vol. 98,1976, pp. 390-402.

Fagan, J. R.; and Fleeter, S: Impeller Flow Field Measurement and Analysis. J. Turbomach., vol. 113, no. 4, 1991, pp. 670-679.

Farge, T. Z.; Johnson, M. W.; and Maksoud, T. M. A.: Tip Leakage in a Centrifugal Impeller. J. Turbomach., vol. 111, no. 2, 1989 , pp. 244-249.

Hah, C.; and Krain, H.: Secondary Flows and Vortex Motion in a High-Efficiency Backswept Impeller at Design and Off-Design Conditions. J. Turbomach., vol. 112, no. 1, 1990, pp. 7-13.

Hanson, K. A. et al.: A Three-Dimensional Unsteady Hot-Wire Technique for the NASA Low-Speed Centrifugal Compressor. NASA TM-105339, 1992

Hathaway, M. D.; Wood, J.R.; and Wasserbauer, C.W.: NASA Low Speed Centrifugal Compressor for 3-D Viscous Code Assessment and Fundamental Flow Physics Research. J. Turbomach,, vol. 114, no. 2, 1992, pp. 295-303.

Joslyn, H. D.; and Dring, R. P.: Surface Indicator and Smoke Flow Visualization Techniques in Rotating Machinery. Heat Transfer and Fluid Flow in Rotating Machinery, W.-J. Yang, ed., Hemisphere Publishing Corp., New York, 1987, pp. 156-169.

Krain, H.; Swirling Impeller Flow. J. Turbomach., vol. 110, no. 1, 1988 , pp. $122-128$

Krain, H.; and Hoffman, W.: Verification of an Impeller Design by Laser Measurements and 3D-Viscous Flow Calculations. ASME Paper 89-GT-159, 1989.

Krain, H.; and Hoffman, W.; Centrifugal Impeller Geometry and its Influence on Secondary Flows. Secondary Flows in Turbomachines, AGARD CP-469, 1990, 17p.

Moore, J.; and Moore, J. G.: A Prediction of 3-D Viscous Flow and Performance of the NASA Low-Speed Centrifugal Compressor. ASME Paper 90-GT-234, 1990.

Nichols, C. E., Jr.: Preparation of Polystyrene Microspheres for Laser Velocimetry in Wind Tunnels. NASA TM-89163, 1987.

Rohne, K. H.; and Banzhaf, M.; Investigation of the Flow at the Exit of an Unshrouded Centrifugal Impeller and Comparison With the "Classical" Jet-Wake Theory. J. Turbomach., vol 113, no. 4, 1990, pp. $654-659$.

Senoo, Y.; Yamaguchi, M.; and Nishi, M.: A Photographic Study of ThreeDimensional Flow in a Radial Compressor. ASME Paper 68-GT-2, 1968.

Sipos, G.: Secondary Flow Loss Distribution in a Radial Compressor with Untwisted Backswept Vanes. J. Turbomach., vol 113, no. 4, 1991, pp. 686-695.

Strazisar, A.J. et al.: Laser Anemometer Measurements in a Transonic Axial-Flow Fan Rotor. NASA TP-2879, 1989.

Wasserbauer, C. W.; and Hathaway, M. D.: Development of the Seeding System Used for Laser Velocimeter Surveys in the NASA Low-Speed Centrifugal Compressor Facility. NASA TM-106046, 1993.

Wood. J. R.; Adam, P. W.; and Buggele, A. E.: NASA Low-Speed Centrifugal Compressor for Fundamental Research. NASA TM-83398, 1983.

Wood, J.R.; Strazisar, A.J.; and Hathaway, M.D.: E/C0-2 Single Transonic Fan Rotor. Test Cases for Computation of Internal Flows in AeroEngine Components, AGARD-AR-275, L. Fottner ed., 1990, 18p. 
Davis Highway, Suite 1204, Arlington, VA 22202-4302, and to the Office of Management and Budget, Paperwork Reduction Project (0704-0188), Washington, DC 20503.

\begin{tabular}{|l|l|l|}
\hline 1. AGENCY USE ONLY (Leave blank) & $\begin{array}{r}\text { 2. REPORT DATE } \\
\text { September } 1993\end{array}$ & $\begin{array}{r}\text { 3. REPORT TYPE AND DATES COVERED } \\
\text { Technical Memorandum }\end{array}$ \\
\hline
\end{tabular}

\section{TITLE AND SUBTITLE}

Experimental and Computational Investigation of the NASA Low-Speed Centrifugal Compressor Flow Field

\section{6. $\operatorname{AUTHOR}(\mathrm{S})$}

Michael D. Hathaway, Randall M. Chriss, Jerry R. Wood, and

Anthony J. Strazisar

7. PERFORMING ORGANIZATION NAME(S) AND ADDRESS(ES)

National Aeronautics and Space Administration

Lewis Research Center

Cleveland, Ohio 44135-3191
5. FUNDING NUMBERS

WU-505-62-52

8. PERFORMING ORGANIZATION REPORT NUMBER

E-7651

10. SPONSORING/MONITORING AGENCY REPORT NUMBER

NASA TM-4481

TR-91-C-052

Propulsion Directorate

U.S. Amy Aviation Systems Command

Cleveland, Ohio 44135-3191

\section{SUPPLEMENTARY NOTES}

Prepared for the 37th International Gas Turbine and Aero Engine Congress and Exposition sponsored by the American Society of Mechanical Engineers, Cologne, Germany, June 1-4, 1992. Michael D. Hathaway, Propulsion Directorate, U.S. Army Aviation Systems Command, Cleveland, Ohio 44135-3191; Randall M. Chriss, J.R. Wood, and Anthony J. Strazisar, NASA Lewis Research Center. Responsible person, Michael D. Hathaway, (216) 433-6250

\begin{tabular}{l|l} 
12a. DISTRIBUTION/AVAILABILITY STATEMENT & 12b. DISTRIBUTION CODE
\end{tabular}

Unclassified - Unlimited

Subject Category 07

13. ABSTRACT (Maximum 200 words)

An experimental and computational investigation of the NASA Lewis Research Center's low-speed centrifugal compressor (LSCC) flow field was conducted using laser anemometry and Dawes' three-dimensional viscous code. The experimental configuration consisted of a backswept impeller followed by a vaneless diffuser. Measurements of the three-dimensional velocity field were acquired at several measurement planes through the compressor. The measurements describe both the throughflow and secondary velocity field along each measurement plane. In several cases the measurements provide details of the flow within the blade boundary layers. Insight into the complex flow physics within centrifugal compressors is provided by the computational fluid dynamics analysis (CFD), and assessment of the CFD predictions is provided by comparison with the measurements. Five-hole probe and hot-wire surveys at the inlet and exit to the impeller as well as surface flow visualization along the impeller blade surfaces provided independent confirmation of the laser measurement technique. The results clearly document the development of the throughflow velocity wake that is characteristic of unshrouded centrifugal compressors.

14. SUBJECT TERMS

5. NUMBER OF PAGES

Centrifugal compressors; Radial turbomachinery; Laser velocimetry

21 OF REPORT

Unclassified
18. SECURITY CLASSIFICATION OF THIS PAGE

Unclassified
19. SECURITY CLASSIFICATION OF ABSTRACT Unclassified 